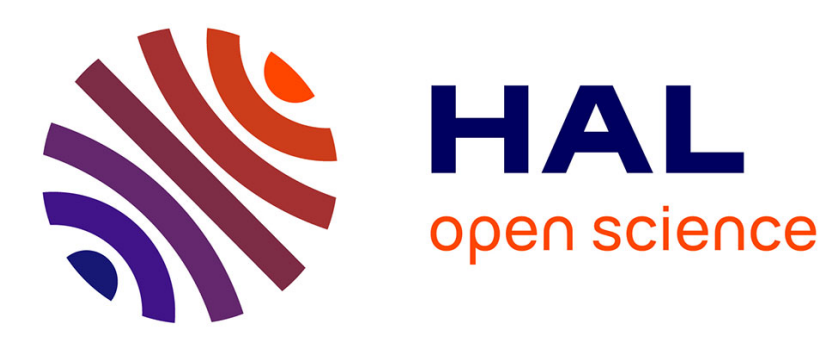

\title{
Robust design optimization in computational mechanics
}

Evangéline Capiez-Lernout, Christian Soize

\section{To cite this version:}

Evangéline Capiez-Lernout, Christian Soize. Robust design optimization in computational mechanics. Journal of Applied Mechanics-Transactions of the Asme, 2008, 75 (2), pp.Article Number: 021001. 10.1115/1.2775493. hal-00686134

\section{HAL Id: hal-00686134 \\ https://hal.science/hal-00686134}

Submitted on 7 Apr 2012

HAL is a multi-disciplinary open access archive for the deposit and dissemination of scientific research documents, whether they are published or not. The documents may come from teaching and research institutions in France or abroad, or from public or private research centers.
L'archive ouverte pluridisciplinaire $\mathbf{H A L}$, est destinée au dépôt et à la diffusion de documents scientifiques de niveau recherche, publiés ou non, émanant des établissements d'enseignement et de recherche français ou étrangers, des laboratoires publics ou privés. 


\title{
Robust design optimization in computational mechanics.
}

\author{
E. Capiez-Lernout ${ }^{1, \star}$, C. Soize ${ }^{2}$ \\ ${ }^{1}$ Université Paris-Est \\ Laboratoire de Mécanique (LaM), \\ EA 2545, \\ 5, Boulevard Descartes, \\ 77454 Marne la Vallée Cedex 2, \\ France
}

Tel: 0033160957798

Fax: 0033160957799

E-mail: Evangeline.Capiez-Lernout@univ-mlv.fr

${ }^{2}$ Université Paris-Est

Laboratoire de Mécanique (LaM),

EA 2545,

5, Boulevard Descartes,

77454 Marne la Vallée Cedex 2,

France

Tel: 0033160957661

Fax: 0033160957799

E-mail: Christian.Soize@univ-mlv.fr

* Corresponding author. 


\begin{abstract}
The motivation of this paper is to propose a methodology for analyzing the robust design optimization problem of complex dynamical systems excited by deterministic loads but taking into account model uncertainties and data uncertainties with an adapted nonparametric probabilistic approach, whereas only data uncertainties are generally considered in the literature by using a parametric probabilistic approach. The possible designs are represented by a numerical finite element model whose design parameters are deterministic and belong to an admissible set. The optimization problem is formulated for the stochastic system as the minimization of a cost function associated to the random response of the stochastic system including the variability of the stochastic system induced by uncertainties and the bias corresponding to the distance of the mean random response to a given target. The gradient and the Hessian of the cost function with respect to the design parameters are explicitly calculated. The complete theory and a numerical application are presented.
\end{abstract}

Key words: Robust design, Model uncertainties, Structural dynamics.

\title{
1 Introduction
}

It is known that the accuracy of the dynamical responses predictions in computational mechanics is mainly related to the level of model and data uncertainties. For this reason, probabilistic models of random uncertainties are implemented in the numerical simulation models in order to improve the predictions in computational dynamics. In this probabilistic context, it is necessary to distinguish the mean dynamical system corresponding to a nominal mechanical model from the stochastic dynamical system corresponding to a more realistic model of the real dynamical system manufactured from the design. These last decades, design optimization has become a major challenge in the industrial technologies such as aerospace, aeronautics, automotive, nuclear industries. In the early works, the design optimization problem was studied from a deterministic point of view, neglecting the presence of uncertainties in the numerical model used for the optimization problem. In this case, the deterministic design optimization problem yields an optimal design whose responses satisfy for the best a given target (performance objective), 
see for instance [1]. This optimal design can then be used in order to construct the stochastic dynamical system for analyzing a posteriori the effects of uncertainties on the dynamical response.

It should be noted that data uncertainties can clearly be taken into account by the parametric probabilistic approach whose stochastic finite element methods (see for instance [2-5]) and other theoretical and numerical methods (see for instance [6,7]) constitute very efficient tools in computational mechanics. Nevertheless, such a parametric probabilistic approach does not allow model uncertainties to be taken into account [8]. More recently, a nonparametric probabilistic approach [9-11] has been proposed to take into account model uncertainties.

These last decades, various researches have been carried out in order to include the effects of uncertainties in the design optimization problem [12] called the robust design. In the context of mechanical engineering, the robust design leads to solve a nonlinear constrained optimization problem with numerical models which are little sensitive to uncertainties in the vicinity of the design point (see for instance [13,14] for the early works and [15-20] for the most recent advances concerning this research area). The main difficulty of such robust design optimization problems concerns the probabilistic model of uncertainties. It should be noted that the relevance of the probabilistic model used is an important factor for the robust design optimization problem. The use of an erroneous probabilistic model yields an erroneous optimal design. However there is no reason for that the responses of the real dynamical system which is manufactured from this erroneous optimal design correspond to the performance objective. Until now, most of the published papers concerning robust design have been carried out in the context of static performances using parametric probabilistic models for modeling data uncertainties in the mechanical system. The robust design in the dynamic field is relatively recent $[18,19]$. It should be noted that the dynamical systems can be very sensitive to model uncertainties and not only sensitive to data uncertainties. Moreover, this sensitivity generally increases with the complexity of the dynamical system. In any case, all the works published until now concern robust design with respect to data uncertainties and not with respect to model uncertainties.

The motivation of this paper is to propose a methodology for analyzing the robust design optimization problem of complex dynamical systems excited by deterministic loads but taking 
into account model uncertainties and data uncertainties with the nonparametric probabilistic approach introduced above, whereas only data uncertainties are generally considered in the literature by using a parametric probabilistic approach. Several experimental validations [21-24] and numerical validations [11,25-27] have proved the capability of the nonparametric probabilistic approach to take into account model uncertainties and data uncertainties. A complete theory concerning the robust design optimization problem of complex dynamical systems excited by deterministic loads is presented in this paper. The nonparametric probabilistic model is used for modeling nonhomogeneous model and data uncertainties. The mean reduced matrix model of the design is constructed by using substructuring techniques (see for instance [28-34]). The design parameters are deterministic and belong to an admissible set. The cost function used to formulate the robust design optimization problem is then defined as a function of the design parameter. Concerning the formulation of the cost function, the performance objective includes not only the target but also the robustness with respect to model uncertainties and data uncertainties. More precisely, the cost function is defined as a linear combination of the normalized variance of the stochastic system related to the variability of the system induced by uncertainties and of the bias corresponding to the distance between the mean random response of the stochastic system and a given target. The norm and the distance used are related to the square integrable norm over a given frequency band of analysis. The gradient and the Hessian of the cost function with respect to the design parameter are algebraically calculated that is useful for improving the performance of the optimization algorithm.

In section 2 , the set of mean reduced matrix models related to the set of all the feasible designs is constructed by using the Benfield and Hruda substructuring technique [30]. In section 3 , the design optimization problem is formulated assuming no uncertainties in the model of the dynamical system. Section 4 is devoted to the implementation of the nonparametric probabilistic approach for model and data uncertainties. Section 5 concerns the construction of the cost function describing the target and the sensitivity of the dynamical system to uncertainties (performance objectives) in order to formulate the robust design optimization problem with respect to model and data uncertainties. Finally, a numerical application is presented in section 6 . 


\section{Mean dynamical system}

The dynamical system is made up of a given master system (a structure) coupled with a subsystem (a substructure) which has to be designed. The dynamical system is assumed to be linear and slightly damped. The equations are discretized by the finite element method and are written in the frequency domain. The frequency band of analysis is denoted by $\mathbb{B}$. It is assumed that the master system has no rigid body displacements and that the subsystem is free with $r$ rigid body modes. Let $\mathbf{p}=\left(p_{1}, \ldots, p_{s}\right)$ be the $\mathbb{C}^{s}$-vector of the design parameters (geometry, elasticity properties, boundary conditions, etc.). The vector of the design parameters belongs to an admissible set $\mathcal{P}$ defined by the set of constraints prescribed by the design. For $\mathbf{p}$ fixed in $\mathcal{P}$ and for $\omega$ fixed in $\mathbb{B}$, the equation of the mean dynamical system is written as

$$
\left(\left[\underline{A}^{1}(\omega)\right]+\left[\underline{A}^{2}(\mathbf{p}, \omega)\right]\right) \underline{\mathbf{u}}(\mathbf{p}, \omega)=\mathbf{f}(\mathbf{p}, \omega),
$$

in which $\underline{\mathbf{u}}(\mathbf{p}, \omega)$ is the $\mathbb{C}^{n}$-vector of the $n$ DOF and $\mathbf{f}(\mathbf{p}, \omega)$ is the $\mathbb{C}^{n}$-vector induced by the external forces. In Eq. (1), the symmetric $n \times n$ complex matrices $\left[\underline{A}^{1}(\omega)\right]$ and $\left[\underline{A}^{2}(\mathbf{p}, \omega)\right]$ are the dynamical stiffness matrices of the master system and of the subsystem. It is assumed that vector $\mathbf{f}(\mathbf{p}, \omega)$ and matrix $\left[\underline{A}^{2}(\mathbf{p}, \omega)\right]$ are affine mappings of the design parameter $\mathbf{p}=\left(p_{1}, \ldots, p_{s}\right)$ and are written as

$$
\begin{aligned}
\mathbf{f}(\mathbf{p}, \omega) & =\mathbf{f}^{0}(\omega)+\sum_{i=1}^{s} p_{i} \mathbf{f}^{i}(\omega), \\
{\left[\underline{A}^{2}(\mathbf{p}, \omega)\right] } & =\left[\underline{A}^{2,0}(\omega)\right]+\sum_{i=1}^{s} p_{i}\left[\underline{A}^{2, i}(\omega)\right] .
\end{aligned}
$$

Note that such an assumption allows a large class of design problems to be studied. For instance, let us consider an Euler beam with rectangular section $S=b h$ and bending inertia $I=b h^{3} / 12=S h^{2} / 12$. It is assumed that $S$ has a fixed value and that the optimization variables are the mass density per unit length $\rho$ and the section height $h$. Then the design parameter can be chosen as $\mathbf{p}=\left(p_{1}, p_{2}\right)$ with $p_{1}=\rho$ and $p_{2}=h^{2}$ which satisfies the linear assumption. Such a linear assumption is useful for optimization algorithm because the gradient of vector $\mathbf{f}(\mathbf{p}, \omega)$ and the gradient of matrix $\left[\underline{A}^{2}(\mathbf{p}, \omega)\right]$ are calculated once and do not depend on $\mathbf{p}$. In addition, the Hessian of $\mathbf{f}(\mathbf{p}, \omega)$ and $\left[\underline{A}^{2}(\mathbf{p}, \omega)\right]$ are zero. It should be noted that the theory pre- 
sented in this paper is also valuable when this linear assumption is removed, but then requires the numerical calculation of the gradient and the Hessian of $\mathbf{f}(\mathbf{p}, \omega)$ and $\left[\underline{A}^{2}(\mathbf{p}, \omega)\right]$ for each $\mathbf{p}$ in $\mathcal{P}$. Let $n_{1}$ and $n_{2}$ be the number of the internal DOF of the master system and the subsystem. Let $n_{\Sigma}$ be the number of coupling interface DOF. We then have $n=n_{1}+n_{\Sigma}+n_{2}$. The bloc decomposition of $\underline{\mathbf{u}}(\mathbf{p}, \omega), \mathbf{f}(\mathbf{p}, \omega),\left[\underline{A}^{1}(\omega)\right]$ and $\left[\underline{A}^{2}(\mathbf{p}, \omega)\right]$ with respect to $n_{1}, n_{\Sigma}$ and $n_{2}$ is given by

$$
\begin{aligned}
& \underline{\mathbf{u}}(\mathbf{p}, \omega)=\left[\begin{array}{l}
\underline{\mathbf{u}}^{1}(\mathbf{p}, \omega) \\
\underline{\mathbf{u}}_{\Sigma}(\mathbf{p}, \omega) \\
\underline{\mathbf{u}}^{2}(\mathbf{p}, \omega)
\end{array}\right] \quad, \quad \mathbf{f}(\mathbf{p}, \omega)=\left[\begin{array}{c}
\mathbf{f}^{1}(\mathbf{p}, \omega) \\
\mathbf{f}_{\Sigma}(\mathbf{p}, \omega) \\
\mathbf{f}^{2}(\mathbf{p}, \omega)
\end{array}\right]
\end{aligned}
$$

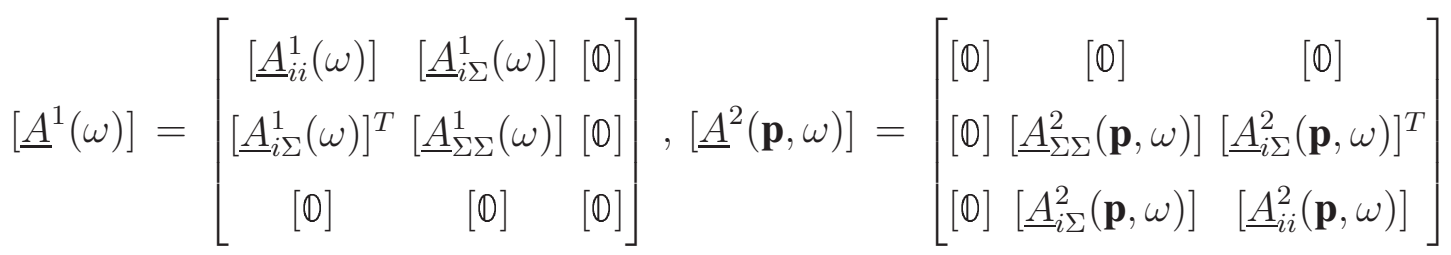

In this paper, the Benfield and Hruda dynamic substructuring method [30] is used and is briefly summarized below (note that any other substructuring method could be used (see for instance [28,29,31-34]). Firstly, the Craig and Bampton method [28] is applied to the master system with $N_{1}$ elastic modes (with fixed coupling interface) stored in the $n_{1} \times N_{1}$ real matrix $\left[\underline{\Phi}^{1}\right]$. The static boundary functions related to this coupling interface are stored in the $n_{1} \times n_{\Sigma}$ real matrix $\left[\underline{S}^{1}\right]$. Secondly, the mean reduced matrices of the master system are assembled with the mean finite element matrices of the subsystem. Thirdly, the submatrix of the coupled system (master system with subsystem) corresponding to the DOF of the subsystem is extracted. The eigenmodes of this submatrix corresponding to the $N_{2}$ lowest eigenfrequencies are stored in the real $\left(n_{\Sigma}+n_{2}\right) \times N_{2}$ matrix $\left[\underline{\Phi}^{2}(\mathbf{p})\right]$. In the robust design optimization context, the probabilistic model of uncertainties must be independent of the value of the design parameter $\mathbf{p}$. This implies that the projection basis for the subsystem must be independent of $\mathbf{p}$. Consequently, a numerical value $\mathbf{p}_{0}$ of $\mathbf{p}$ is chosen as an initial design value. Let $V_{N_{2}}$ be the subspace of $\mathbb{R}^{n_{\Sigma}+n_{2}}$ spanned by the $N_{2}$ columns of $\left[\underline{\Phi}^{2}\left(\mathbf{p}_{0}\right)\right]$. The value of $N_{2}$ must be chosen such that $\left(\underline{\mathbf{u}}_{\Sigma}(\mathbf{p}, \omega), \underline{\mathbf{u}}^{2}(\mathbf{p}, \omega)\right)$ belongs to $V_{N_{2}}$ for all $\mathbf{p}$ in $\mathcal{P}$ for a given tolerance of this approximation. It should be noted that $N_{2}$ can not be selected a priori, but has to be defined during the computation studying the convergence with respect to $N_{2}$. Clearly, $V_{N_{2}}$ converges to $\mathbb{R}^{n_{\Sigma}+n_{2}}$ when $N_{2}$ goes to $n_{2}+n_{\Sigma}$, for all $\mathbf{p}$ in $\mathcal{P}$. Nevertheless, the reduction will be efficient if $N_{2} \ll n_{2}+n_{\Sigma}$. It will be the case 
if the set $\mathcal{P}$ of the possible designs is not too "big" and for $n_{2}+n_{\Sigma}$ very large. If $n_{2}+n_{\Sigma}$ is small (for instance a few dozens of degrees of freedom), we will have $N_{2} \simeq n_{2}+n_{\Sigma}$ but it is not a difficulty. If $n_{2}+n_{\Sigma}$ is large (for instance several thousands or ten thousands of degrees of freedom) and if the convergence is not obtained for $N_{2} \ll n_{2}+n_{\Sigma}$, this means that the projection basis has to be constructed for each $\mathbf{p}$ in $\mathcal{P}$. For such a case, all the developments presented in this paper can be used except the explicit calculation of the gradient and the Hessian of the cost function which then have to be numerically calculated (if used in the optimization algorithm). Finally, the projection basis corresponding to the Benfield and Hruda dynamic substructuring method is then written as

$$
\left[\begin{array}{l}
\underline{\mathbf{u}}^{1}(\mathbf{p}, \omega) \\
\underline{\mathbf{u}}_{\Sigma}(\mathbf{p}, \omega) \\
\underline{\mathbf{u}}^{2}(\mathbf{p}, \omega)
\end{array}\right]=\left[\underline{H}\left(\mathbf{p}_{0}\right)\right]\left[\begin{array}{l}
\underline{\mathbf{q}}^{1}(\mathbf{p}, \omega) \\
\underline{\mathbf{q}}^{2}(\mathbf{p}, \omega)
\end{array}\right] \quad, \quad\left[\underline{H}\left(\mathbf{p}_{0}\right)\right]=\left[\begin{array}{cc}
{\left[\underline{\Phi}^{1}\right]} & {\left[\underline{S}^{1}\right]\left[\underline{\Phi}_{\Sigma}^{2}\left(\mathbf{p}_{0}\right)\right]} \\
{[\mathbb{0}]} & {\left[\underline{\Phi}_{\Sigma}^{2}\left(\mathbf{p}_{0}\right)\right]} \\
{[\mathbb{0}]} & {\left[\underline{\Phi}_{i}^{2}\left(\mathbf{p}_{0}\right)\right]}
\end{array}\right]
$$

in which the matrices $\left[\Phi_{\Sigma}^{2}\left(\mathbf{p}_{0}\right)\right]$ and $\left[\underline{\Phi}_{i}^{2}\left(\mathbf{p}_{0}\right)\right]$ correspond to the bloc decomposition of matrix $\left[\underline{\Phi}^{2}\left(\mathbf{p}_{0}\right)\right]$ with respect to the $n_{\Sigma}$ coupling interface DOF and the $n_{2}$ internal DOF of the subsystem. Projecting Eq. (1) in using Eq. (6) yields the mean reduced matrix equation

$$
\left(\left[\underline{A}_{\mathrm{red}}^{1}(\omega)\right]+\left[\underline{A}_{\mathrm{red}}^{2}(\mathbf{p}, \omega)\right]\right)\left[\begin{array}{l}
\underline{\mathbf{q}}^{1}(\mathbf{p}, \omega) \\
\underline{\mathbf{q}}^{2}(\mathbf{p}, \omega)
\end{array}\right]=\mathbf{f}_{\mathrm{red}}(\mathbf{p}, \omega)
$$

in which $\mathbf{f}_{\text {red }}(\mathbf{p}, \omega)=\left[\underline{H}\left(\mathbf{p}_{0}\right)\right]^{T} \mathbf{f}(\mathbf{p}, \omega)$ and where $\left[\underline{A}_{\text {red }}^{1}(\omega)\right]$ and $\left[\underline{A}_{\mathrm{red}}^{2}(\mathbf{p}, \omega)\right]$ are the symmetric $N \times N$ complex matrices in which $N=N_{1}+N_{2}$ using the bloc decomposition with respect to the $N_{1}$ and $N_{2}$ reduced coordinates. Note that $\left[\underline{A}_{\mathrm{red}}^{1}(\omega)\right]$ is a full matrix and that $\left[\underline{A}_{\mathrm{red}}^{2}(\mathbf{p}, \omega)\right]$ is written as

$$
\left[\underline{A}_{\mathrm{red}}^{2}(\mathbf{p}, \omega)\right]=\left[\begin{array}{lc}
{[\mathbb{Q}]} & {[\mathbb{Q}]} \\
{[\mathbb{Q}]} & {\left[\underline{A}_{s}^{2}(\mathbf{p}, \omega)\right]}
\end{array}\right]
$$

in which

$$
\left[\underline{A}_{s}^{2}(\mathbf{p}, \omega)\right]=\left[[\mathbb{\mathbb { O }}]^{T}\left[\underline{\Phi}^{2}\left(\mathbf{p}_{0}\right)\right]^{T}\right]\left[\underline{A}^{2}(\mathbf{p}, \omega)\right]\left[\begin{array}{c}
{[\mathbb{Q}]} \\
{\left[\underline{\Phi}^{2}\left(\mathbf{p}_{0}\right)\right]}
\end{array}\right] .
$$




\section{Formulation for the design optimization problem with a numerical model with no un- certainties}

In this Section, we remind a formulation to solve the design optimization problem assuming that there is no uncertainties. This formulation will be used to compare the solution of this deterministic design optimization problem with the robust design optimization solution presented in Section 5. Let $\underline{\mathbf{w}}(\mathbf{p}, \omega)$ be the vector in $\mathbb{C}^{k}$ of the observations of the mean dynamical system, defined as a function of the displacement vector $\underline{\mathbf{u}}(\mathbf{p}, \omega)$ such that

$$
\underline{\mathbf{w}}(\mathbf{p}, \omega)=\mathbf{b}_{\omega}(\underline{\mathbf{u}}(\mathbf{p}, \omega))
$$

where $\mathbf{b}_{\omega}$ is a given function from $\mathbb{C}^{n}$ into $\mathbb{C}^{k}$ depending on the frequency $\omega$. The performance objectives for the observations in the frequency band $\mathbb{B}_{1} \subset \mathbb{B}$ will be defined as the "target". This target is then represented by the function $\omega \mapsto \mathbf{g}(\omega)$ from $\mathbb{B}_{1}$ into $\mathbb{C}^{k}$. The cost function $\underline{j}(\mathbf{p})$ is formulated as a distance between the target $\mathbf{g}$ and the observation $\underline{\mathbf{w}}(\mathbf{p}, \cdot)$. It is a function of design parameter $\mathbf{p}$ and is written as

$$
\underline{j}(\mathbf{p})=\frac{\|\underline{\mathbf{w}}(\mathbf{p}, \cdot)-\mathbf{g}\|_{\mathbb{B}_{1}}^{2}}{\|\mathbf{g}\|_{\mathbb{B}_{1}}^{2}},
$$

in which $\|\mathbf{g}\|_{\mathbb{B}_{1}}^{2}=\int_{\mathbb{B}_{1}}\|\mathbf{g}(\omega)\|^{2} d \omega$ with $\|\mathbf{g}(\omega)\|$ the hermitian norm of $\mathbf{g}(\omega)$. The design optimization problem is formulated as the minimization of the cost function $\underline{j}(\mathbf{p})$ with respect to the design parameter $\mathbf{p}$ in the admissible set $\mathcal{P}$ : find $\mathbf{p}^{\mathrm{D}}$ in $\mathcal{P}$ such that $\underline{j}\left(\mathbf{p}^{\mathrm{D}}\right) \leq \underline{j}(\mathbf{p})$ for all $\mathbf{p}$ in $\mathcal{P}$. Such an optimization problem can be solved numerically by using the sequential quadratic optimization algorithm $[35,36]$.

\section{Stochastic dynamical system with model and data uncertainties}

As explained in the Introduction, the robust design optimization problem is formulated with respect to the model uncertainties and data uncertainties existing in the mean model of the dynamical system. In this Section, we then introduce this nonparametric probabilistic approach of uncertainties [9-11]. It is assumed that the mean model of the master system and subsystem 
contain model uncertainties and data uncertainties. The level of uncertainties of these two systems is a priori different and will be then characterized by different values of the dispersion parameters defined below.

Let $\left[\underline{M}_{\mathrm{red}}^{1}\right],\left[\underline{D}_{\mathrm{red}}^{1}\right],\left[\underline{K}_{\mathrm{red}}^{1}\right]$ and $\left[\underline{M}_{s}^{2}(\mathbf{p})\right],\left[\underline{D}_{s}^{2}(\mathbf{p})\right],\left[\underline{K}_{s}^{2}(\mathbf{p})\right]$ be the mean reduced mass, damping, stiffness matrices of the mean master system and of the mean subsystem respectively. The dynamic stiffness reduced matrices are such that $\left[\underline{A}_{\mathrm{red}}^{1}(\omega)\right]=-\omega^{2}\left[\underline{M}_{\mathrm{red}}^{1}\right]+i \omega\left[\underline{D}_{\mathrm{red}}^{1}\right]+\left[\underline{K}_{\mathrm{red}}^{1}\right]$ and $\left[\underline{A}_{s}^{2}(\mathbf{p}, \omega)\right]=-\omega^{2}\left[\underline{M}_{s}^{2}(\mathbf{p})\right]+i \omega\left[\underline{D}_{s}^{2}(\mathbf{p})\right]+\left[\underline{K}_{s}^{2}(\mathbf{p})\right]$. The matrices $\left[\underline{M}_{\mathrm{red}}^{1}\right],\left[\underline{D}_{\mathrm{red}}^{1}\right],\left[\underline{K}_{\mathrm{red}}^{1}\right]$ are positive symmetric $N \times N$ matrices whose rank is $N_{1}=N-n_{2}$ (which is assumed to be positive) whereas $\left[\underline{M}_{S}^{2}(\mathbf{p})\right]$ is a positive-definite symmetric $N_{2} \times N_{2}$ matrix and $\left[\underline{D}_{s}^{2}(\mathbf{p})\right]$, $\left[\underline{K}_{s}^{2}(\mathbf{p})\right]$ are positive symmetric $N_{2} \times N_{2}$ matrices whose rank is $N_{2}-r$. The methodology of the nonparametric probabilistic approach consists in replacing the matrices $\left[\underline{M}_{\mathrm{red}}^{1}\right],\left[\underline{D}_{\mathrm{red}}^{1}\right]$, $\left[\underline{K}_{\mathrm{red}}^{1}\right]$ and $\left[\underline{M}_{s}^{2}(\mathbf{p})\right],\left[\underline{D}_{s}^{2}(\mathbf{p})\right],\left[\underline{K}_{s}^{2}(\mathbf{p})\right]$ by the random matrices $\left[\mathbf{M}_{\mathrm{red}}^{1}\right],\left[\mathbf{D}_{\mathrm{red}}^{1}\right],\left[\mathbf{K}_{\mathrm{red}}^{1}\right]$ and $\left[\mathbf{M}_{s}^{2}(\mathbf{p})\right]$, $\left[\mathbf{D}_{s}^{2}(\mathbf{p})\right],\left[\mathbf{K}_{s}^{2}(\mathbf{p})\right]$ such that $\mathcal{E}\left\{\left[\mathbf{M}_{\text {red }}^{1}\right]\right\}=\left[\underline{M}_{\mathrm{red}}^{1}\right], \mathcal{E}\left\{\left[\mathbf{D}_{\text {red }}^{1}\right]\right\}=\left[\underline{D}_{\text {red }}^{1}\right], \mathcal{E}\left\{\left[\mathbf{K}_{\text {red }}^{1}\right]\right\}=\left[\underline{K}_{\text {red }}^{1}\right]$ and $\mathcal{E}\left\{\left[\mathbf{M}_{s}^{2}(\mathbf{p})\right]\right\}=\left[\underline{M}_{s}^{2}(\mathbf{p})\right], \mathcal{E}\left\{\left[\mathbf{D}_{s}^{2}(\mathbf{p})\right]\right\}=\left[\underline{D}_{s}^{2}(\mathbf{p})\right], \mathcal{E}\left\{\left[\mathbf{K}_{s}^{2}(\mathbf{p})\right]\right\}=\left[\underline{K}_{s}^{2}(\mathbf{p})\right]$ in which $\mathcal{E}$ is the mathematical expectation.

The probability model for each one of these random matrices is briefly recalled below. Let $\left[\underline{E}^{i}(\mathbf{p})\right], i=\{1,2\}$ be the positive symmetric $n \times n$ real matrix of rank $m$ representing one of the matrices of the set $\left\{\left[\underline{M}_{\mathrm{red}}^{1}\right],\left[\underline{D}_{\mathrm{red}}^{1}\right],\left[\underline{K}_{\mathrm{red}}^{1}\right]\right\}$ when $i=1$ or of the set $\left\{\left[\underline{M}_{s}^{2}(\mathbf{p})\right],\left[\underline{D}_{s}^{2}(\mathbf{p})\right]\right.$, $\left.\left[\underline{K}_{s}^{2}(\mathbf{p})\right]\right\}$ when $i=2$. Using the nonparametric probabilistic approach, the matrix $\left[\underline{E}^{i}(\mathbf{p})\right]$ is replaced by the random matrix $\left[\mathbf{E}^{i}(\mathbf{p})\right]$ such that

$$
\left[\mathbf{E}^{i}(\mathbf{p})\right]=\left[\underline{L}_{E}^{i}(\mathbf{p})\right]^{T}\left[\mathbf{G}_{E}^{i}\right]\left[\underline{L}_{E}^{i}(\mathbf{p})\right]
$$

in which $\left[\underline{L}_{E}^{i}(\mathbf{p})\right]$ is a $m \times n$ rectangular real matrix such that $\left[\underline{E}^{i}(\mathbf{p})\right]=\left[\underline{L}_{E}^{i}(\mathbf{p})\right]^{T}\left[\underline{L}_{E}^{i}(\mathbf{p})\right]$ and where $\left[\mathbf{G}_{E}^{i}\right]$ is a random matrix with value in the set of all the positive-definite symmetric $m \times m$ matrices. The probability model of random matrix $\left[\mathbf{G}_{E}^{i}\right]$ is constructed by using the maximum entropy principle with the available information. The dispersion of the random matrix $\left[\mathbf{G}_{E}^{i}\right]$ is controlled by one real positive parameter $\delta_{E}^{i}$ called the dispersion parameter. This means that the dispersion parameters related to random matrices $\left[\mathbf{G}_{M}^{1}\right],\left[\mathbf{G}_{D}^{1}\right],\left[\mathbf{G}_{K}^{1}\right]$ and $\left[\mathbf{G}_{M}^{2}\right],\left[\mathbf{G}_{D}^{2}\right],\left[\mathbf{G}_{K}^{2}\right]$ are $\delta_{M}^{1}, \delta_{D}^{1}, \delta_{K}^{1}$ and $\delta_{M}^{2}, \delta_{D}^{2}, \delta_{K}^{2}$. It should be noted that as a result of this theory, these six random 
matrices are independent random matrices. All the details concerning the construction of this probability model can be found in [9-11]. The algebraic representation of random matrix $\left[\mathbf{G}_{E}^{i}\right]$ adapted to the Monte Carlo numerical simulation is briefly recalled. Random matrix $\left[\mathbf{G}_{E}^{i}\right]$ is written as $\left[\mathbf{G}_{E}^{i}\right]=\left[\mathbf{L}_{G_{E}^{i}}\right]^{T}\left[\mathbf{L}_{G_{E}^{i}}\right]$ in which $\left[\mathbf{L}_{G_{E}^{i}}\right]$ is a $m \times m$ real upper triangular random matrix such that

(1) random variables $\left\{\left[\mathbf{L}_{G_{E}^{i}}\right]_{j j^{\prime}}, j \leq j^{\prime}\right\}$ are independent;

(2) for $j<j^{\prime}$, real-valued random variable $\left[\mathbf{L}_{G_{E}^{i}}\right]_{j j^{\prime}}$ can be written as $\left[\mathbf{L}_{G_{E}^{i}}\right]_{j j^{\prime}}=\sigma_{m} U_{j j^{\prime}}$ in which $\sigma_{m}=\delta_{E}^{i}(m+1)^{-1 / 2}$ and where $U_{j j^{\prime}}$ is a real-valued Gaussian random variable with zero mean and variance equal to 1 ;

(3) for $j=j^{\prime}$, positive-valued random variable $\left[\mathbf{L}_{G_{E}^{i}}\right]_{j j}$ can be written as $\left[\mathbf{L}_{G_{E}^{i}}\right]_{j j}=\sigma_{m} \sqrt{2 V_{j}}$ in which $\sigma_{m}$ is defined above and where $V_{j}$ is a positive-valued gamma random variable whose probability density function $p_{V_{j}}(v)$ with respect to $d v$ is written as

$$
p_{V_{j}}(v)=\mathbb{1}_{\mathbb{R}^{+}}(v) \frac{1}{\Gamma\left(\frac{m+1}{2\left(\delta_{E}^{i}\right)^{2}}+\frac{1-j}{2}\right)} v^{\frac{m+1}{2\left(\delta_{E}^{i}\right)^{2}}-\frac{1+j}{2}} e^{-v}
$$

In coherence with the notation of Section 2 , let $\mathbf{U}(\mathbf{p}, \omega)$ be the $\mathbb{C}^{n}$-valued random vector of the displacement whose bloc decomposition, (similar to Eq. (4)), is written as $\mathbf{U}(\mathbf{p}, \omega)=$ $\left(\mathbf{U}^{1}(\mathbf{p}, \omega), \mathbf{U}_{\Sigma}(\mathbf{p}, \omega), \mathbf{U}^{2}(\mathbf{p}, \omega)\right)$. Then the equations of the stochastic reduced system corresponding to the nonparametric probabilistic model of uncertainties are

$$
\left[\begin{array}{c}
\mathbf{U}^{1}(\mathbf{p}, \omega) \\
\mathbf{U}_{\Sigma}(\mathbf{p}, \omega) \\
\mathbf{U}^{2}(\mathbf{p}, \omega)
\end{array}\right]=\left[\underline{H}\left(\mathbf{p}_{0}\right)\right]\left[\begin{array}{c}
\mathbf{Q}^{1}(\mathbf{p}, \omega) \\
\mathbf{Q}^{2}(\mathbf{p}, \omega)
\end{array}\right]
$$

where $\mathbf{Q}(\mathbf{p}, \omega)=\left(\mathbf{Q}^{1}(\mathbf{p}, \omega), \mathbf{Q}^{2}(\mathbf{p}, \omega)\right)$ is the $\mathbb{C}^{N}$-valued random vector of the generalized coordinates, solution of the random matrix equation that

$$
\left(\left[\mathbf{A}_{\text {red }}^{1}(\omega)\right]+\left[\mathbf{A}_{\text {red }}^{2}(\mathbf{p}, \omega)\right]\right) \mathbf{Q}(\mathbf{p}, \omega)=\mathbf{f}_{\text {red }}(\mathbf{p}, \omega)
$$

in which the matrix $\left[\mathbf{A}_{\text {red }}^{1}(\omega)\right]$ is such that $\left[\mathbf{A}_{\text {red }}^{1}(\omega)\right]=-\omega^{2}\left[\mathbf{M}_{\text {red }}^{1}\right]+i \omega\left[\mathbf{D}_{\text {red }}^{1}\right]+\left[\mathbf{K}_{\text {red }}^{1}\right]$ and where 
the matrix $\left[\mathbf{A}_{\text {red }}^{2}(\mathbf{p}, \omega)\right]$ is such that

$$
\left[\mathbf{A}_{\mathrm{red}}^{2}(\mathbf{p}, \omega)\right]=\left[\begin{array}{lc}
{[\mathbb{\mathbb { Q }}]} & {[\mathbb{Q}]} \\
{[\mathbb{\mathbb { Q }}]} & {\left[\mathbf{A}_{s}^{2}(\mathbf{p}, \omega)\right]}
\end{array}\right],
$$

with

$$
\left[\mathbf{A}_{s}^{2}(\mathbf{p}, \omega)\right]=-\omega^{2}\left[\mathbf{M}_{s}^{2}(\mathbf{p})\right]+i \omega\left[\mathbf{D}_{s}^{2}(\mathbf{p})\right]+\left[\mathbf{K}_{s}^{2}(\mathbf{p})\right]
$$

\section{Formulation of the robust design optimization problem with respect to model uncer- tainties and data uncertainties}

In this Section, the robust design optimization problem is formulated with respect to model uncertainties and data uncertainties using the nonparametric probabilistic approach described in Section 4 . The robust design optimization problem deals with the minimization of a cost function with respect to the design parameter. The cost function is constructed with an uncertain numerical model. Contrary to the design optimization problem described in Section 3, the cost function is not defined for the performance of the mean dynamical system but is defined with respect to the performance of the stochastic dynamical system representing the real manufactured system. For the robust problem, the performance objectives are double: (1) minimizing the distance between the mean value of the stochastic observation and the target and (2) minimizing the sensitivity of the stochastic observation with respect to model uncertainties and data uncertainties. The solution of this robust design optimization problem yields an optimal value of the design parameter which corresponds to an optimal dynamical system from which the real manufactured system fulfills the performance objectives.

In coherence with the notation introduced in Section 3 , let $\mathbf{W}(\mathbf{p}, \omega)$ be the $\mathbb{C}^{k}$-valued random variable modeling the random observation of the stochastic dynamical system. It is defined as a function of the random displacement vector $\mathbf{U}(\mathbf{p}, \omega)$ such that

$$
\mathbf{W}(\mathbf{p}, \omega)=\mathbf{b}_{\omega}(\mathbf{U}(\mathbf{p}, \omega))
$$


in which $\mathbf{b}_{\omega}$ is the function introduced in Eq. (10). The cost function is then defined by

$$
j(\mathbf{p}, \alpha)=\frac{1}{\|\mathbf{g}\|_{\mathbb{B}_{1}}^{2}}\left(\alpha\|\boldsymbol{\mu}(\mathbf{p}, \cdot)-\mathbf{g}\|_{\mathbb{B}_{1}}^{2}+(1-\alpha) \sigma^{2}(\mathbf{p})\right),
$$

in which $\boldsymbol{\mu}(\mathbf{p}, \omega)$ is the mean value of random vector $\mathbf{W}(\mathbf{p}, \omega)$ such that $\boldsymbol{\mu}(\mathbf{p}, \omega)=\mathcal{E}\{\mathbf{W}(\mathbf{p}, \omega)\}$ and where $\sigma^{2}(\mathbf{p})=\mathcal{E}\left\{\|\mathbf{W}(\mathbf{p}, \cdot)-\boldsymbol{\mu}(\mathbf{p}, \cdot)\|_{\mathbb{B}_{1}}^{2}\right\}$. In Eq. (19), the scalar $\alpha$ belonging to $\left.] 0,1 / 2\right]$ is the weighting factor which has to be adjusted in order to favorize the robustness objective performance (2) with respect to the target objective performance (1). It should be noted that cost function $j(\mathbf{p}, \alpha)$ can be rewritten as

$$
j(\mathbf{p}, \alpha)=\alpha j_{1}(\mathbf{p})+(1-2 \alpha) j_{2}(\mathbf{p})
$$

in which $j_{1}(\mathbf{p})=\|\mathbf{g}\|_{\mathbb{B}_{1}}^{-2} \mathcal{E}\left\{\|\mathbf{W}(\mathbf{p}, \cdot)-\mathbf{g}\|_{\mathbb{B}_{1}}^{2}\right\}$ and where $j_{2}(\mathbf{p})=\|\mathbf{g}\|_{\mathbb{B}_{1}}^{-2} \sigma^{2}(\mathbf{p})$. Note that $j_{1}(\mathbf{p})$ is related to a distance between the stochastic observation and the target and allows the target performance objective (1) and the robustness performance objective (2) to be simultaneously achieved with equal weight. The quantity $j_{2}(\mathbf{p})$ is a penalty term for favorizing the robustness performance objective (2) when $\alpha$ decreases.

The robust design optimization problem is formulated as a multiobjective optimization problem which consists to minimize the cost function $\mathbf{p} \mapsto j(\mathbf{p}, \alpha)$ with respect to the admissible set $\mathcal{P}$ of the design parameter $\mathbf{p}$. For given dispersion parameters $\delta_{M}^{1}, \delta_{D}^{1}, \delta_{K}^{1}$ and $\delta_{M}^{2}, \delta_{D}^{2}, \delta_{K}^{2}$ and for a given value of $\alpha \in] 0,1 / 2]$, the robust design optimization problem is written as : find $\mathbf{p}^{\mathrm{RD}}$ in $\mathcal{P}$ such that

$$
j\left(\mathbf{p}^{\mathrm{RD}}, \alpha\right) \leq j(\mathbf{p}, \alpha), \text { for all } \mathbf{p} \text { in } \mathcal{P}
$$

The value of the weighting factor $\alpha$ characterizes the importance of each performance objective with respect to the other one and is adjusted in order to obtain the better solution. Since the paper deals with robust design optimization, the case for which the target objective performance (1) would be favorized with respect to the robust objective performance (2) is not considered. For this reason, the weighting factor is chosen such that $\alpha \leq 1 / 2$. When $\alpha=1 / 2$, the weight of the performance objectives (1) and (2) are the same. For small values of $\alpha$, the performance objective related to the robustness with respect to model and data uncertainties becomes more important with respect to the performance objective related to the target. In the 
formulation of the cost function $j(\mathbf{p}, \alpha)$ given by Eq. (19), a target has been introduced. Then the cost function given by Eq. (19) is different from most of the formulations encountered in the literature $[15,18,19]$. In addition, since the normalisation does not change the optimization problem, it should be noted that the formulation used is coherent with the usual formulation of the robust design optimization problem [18-20] when the target is not taken into account $(\mathbf{g}=0)$ and for the mono-dimensional case $(k=1)$. Note that the definition of the robust design optimization problem is coherent with respect to the deterministic design optimization problem given in Section 3. Since the value $\alpha=0$ is excluded, the formulation for robust design optimization is coherent with the formulation of design optimization when the stochastic dynamical system tends to be deterministic, that is to say when $\lim _{\|\boldsymbol{\delta}\| \mapsto \mathbf{0}} \mathbf{p}^{\mathrm{RD}}=\mathbf{p}^{\mathrm{D}}$ in which $\boldsymbol{\delta}=\left(\delta_{M}^{1}, \delta_{D}^{1}, \delta_{K}^{1}, \delta_{M}^{2}, \delta_{D}^{2}, \delta_{K}^{2}\right)$. Finally, the robust design optimization problem is solved by using the sequential quadratic optimization algorithm [35,36] coupled with the Monte Carlo numerical simulation. In addition, it should be noted that the random germs of the random matrices do not depend on the design parameter $\mathbf{p}$. Consequently, the gradient and the Hessian can algebraically be constructed (see Appendix), that improves the precision of the optimization algorithm.

\section{Numerical application}

The objective of this application is the robust design optimization of dynamic absorbers in order to reduce the vibration level of a heterogeneous dynamical system in a given narrowfrequency band and for a given deterministic excitation. The heterogeneous dynamical system is constituted of a homogeneous plate coupled with lumped masses and oscillators. Only the vibration level of the homogeneous plate has to be reduced. The dynamic absorbers are identical and each one is constituted of oscillators in parallel at different eigenfrequencies. Two cases are investigated. The first case concerns robust design optimization with respect to a single design parameter. A more complicated case involving a multidimensional design parameter is then considered. 


\subsection{Mean finite element model of the dynamical system}

The dynamical system is constituted of a master system corresponding to the heterogeneous dynamical system and of a subsystem made up of the dynamic absorbers. The frequency band of interest is $[350,750] \mathrm{Hz}$ but the frequency band of analysis for optimal design is $[500,600] \mathrm{Hz}$. Consequently, the target will be specified in this frequency band in order to optimize the absorbers in this band.

The mean master system is the heterogeneous system made up of a plate with two attached lumped masses, one attached spring and 51 attached single DOF linear oscillators. The plate is a thin plate in bending mode and is located in the plane $(O x, O y)$ of a cartesian coordinate system $(O x y z)$. The out-plane displacements are only considered. The plate is made of a homogeneous, isotropic elastic material with mass density $7800 \mathrm{Kg} \times \mathrm{m}^{-3}$, Poisson ratio 0.29 , Young modulus $2 \times 10^{11} N \times \mathrm{m}^{-2}$. The plate has constant thickness $0.4 \times 10^{-3} \mathrm{~m}$, length $0.5 \mathrm{~m}$ and width $0.4 \mathrm{~m}$. The plate is simply supported on three edges and is free on the fourth edge corresponding to $y=0$. The mean finite element model of the plate is constituted of 2000 bending plate elements (with 4 nodes) and is shown in Fig. 1. A damping model is added to the plate corresponding to a hysteretic model with a mean loss factor 0.02 . The two lumped masses have mass $4 \mathrm{Kg}$ and $1 \mathrm{Kg}$, located at points $(0.15,0.15,0)$ and $(0.2,0,0)$ respectively (see Fig. 1). The attached spring has stiffness $2.388 \times 10^{11} \mathrm{~N} \times \mathrm{m}^{-1}$ and is located at point $(0.06,0.23,0)$ (see Fig. 1). The attached oscillators are grouped by sets of 3 oscillators (see Fig. 1). The eigenfrequencies of these oscillators are in the band $[350,750] \mathrm{Hz}$.

The mean subsystem to be optimized is constituted of 9 identical vibration absorbers, each one being made up of 5 single DOF linear oscillators in parallel (see Fig. 1). The eigenfrequencies of the 5 oscillators are fixed, are equal to $560,565,570,575,580 \mathrm{~Hz}$ and have been chosen to form an absorber around the main resonance occuring at $571 \mathrm{~Hz}$ in the narrow-frequency band $[500,600] \mathrm{Hz}$. Note that these values have been deduced from a sensitivity analysis performed with the mean model in order to obtain a significant reduction of the vibration levels. The critical damping rate is the same for the 5 oscillators and is 0.01 . In a first case, it is assumed that the five oscillators of a vibration absorber have the same mass $m$ which has to be 
optimized. The total mass of the subsystem is then defined by $m=45 \mathrm{~m}$. The design parameter is $\mathrm{m}$. In a second case, it is assumed that the mass of each oscillator constituting a vibration absorber can be different. Denoting as $m_{i}$ the mass of the oscillator number $i$ of each vibration absorber constituting the subsystem, the design parameter is the $\mathbb{R}^{5}$-vector $m$ in which $\mathrm{m}=\left(9 m_{1}, 9 m_{2}, 9 m_{3}, 9 m_{4}, 9 m_{5}\right)$. Since the eigenfrequency and the critical damping of the oscillators of the vibration absorbers are fixed, the mass, damping and stiffness matrices of the subsystem are affine functions of the design parameter $\mathrm{m}$. In the present case, the excitation does not depend on $\mathrm{m}$.

The finite element model of the mean dynamical system (master system coupled with the five vibration absorbers) is thus constituted of $n=6106$ DOF with $n_{1}=6052$ internal DOF of the mean master system, $n_{2}=45$ internal DOF for the mean subsystem and $n_{\Sigma}=9$ coupling interface DOF. The mean dynamical system is submitted to a given deterministic unit transverse load constant in frequency band $[5,1200] \mathrm{Hz}$ with amplitude 1 (see Fig. 1). The observation chosen for the dynamic analysis is the signal energy related to the out-plane accelerations of the plate. Consequently, the real-valued function $b_{\omega}$ introduced in Eq. (9) is such that $\underline{w}(\mathbf{p}, \omega)=$ $b_{\omega}(\underline{\mathbf{u}}(\mathbf{p}, \omega))=\omega^{2}\left\|\underline{\mathbf{u}}^{\text {plate }}(\mathbf{p}, \omega)\right\|$ in which $\underline{\mathbf{u}}^{\text {plate }}(\mathbf{p}, \omega)$ is the complex vector constituted of the 1960 out-plane displacements of the plate.

\subsection{Reference solution for the master system}

The reference observation in the master system is $\underline{w}^{\operatorname{master}}(\omega)=\omega^{2}\left\|\underline{\mathbf{u}}_{\text {unc }}^{\text {plate }}(\mathbf{p}, \omega)\right\|$, in which $\underline{\mathbf{u}}_{\text {unc }}^{\text {plate }}$ is the plate response corresponding to the mean model of the master system (uncoupled with the absorbers of the subsystem). Figure 2 displays the graph of $\omega \mapsto 20 \log _{10}\left(\underline{w}^{\text {master }}(\omega)\right)$. In Fig. 2, it can be seen that the level of the reference solution for the mean master system is lower than $77.5 \mathrm{~dB}$ in the frequency band $[350,750] \mathrm{Hz}$ except for one single peak whose resonance occurs at $571 \mathrm{~Hz}$ with level $80.5 \mathrm{~dB}$, i.e. $3 \mathrm{~dB}$ more. 


\subsection{Estimation of the numerical parameters for the robust design optimization problem}

Let $\left\{W^{\text {master }}\left(\theta_{j}, \omega\right), j=1, \ldots, n_{s}\right\}$ be the $n_{s}$ independent realisations of random variable $W^{\text {master }}(\omega)$. The robust optimization problem needs to solve the stochastic reduced equation Eq. (15). The numerical parameters are then the dimension $N$ of the reduced dynamical system and the number $n_{s}$ of realizations used in the Monte Carlo numerical simulation. Therefore a convergence analysis has to be performed with respect to $N$ and $n_{s}$ for the stochastic reduced system. The computation is performed for the dispersion parameters of the master system such that $\delta_{M}^{1}=\delta_{D}^{1}=\delta_{K}^{1}=0.05$. A stochastic convergence analysis with $N_{2}=n_{2}+n_{\Sigma}=54$ is carried out in order to define the number $N_{1}$ of modes to be kept in the modal reduction and the number $n_{s}$ of realizations. The mean-square convergence is analyzed by studying the function $\left(n_{s}, N_{1}\right) \mapsto \operatorname{Conv}\left(n_{s}, N_{1}\right)$ defined by

$$
\left.\operatorname{Conv}\left(n_{s}, N_{1}\right)=\left\{\frac{1}{n_{s}} \sum_{j=1}^{n_{s}} \int_{\mathbb{B}} \| W^{\operatorname{master}}\left(\theta_{j}, \omega\right)\right) \|^{2} d \omega\right\}^{1 / 2}
$$

in which $W^{\text {master }}\left(\theta_{j}, \omega\right)$ is calculated with a reduced model of dimension $N=N_{1}+N_{2}$. Fig. 3 displays the graph of $n_{s} \mapsto 20 \log _{10}\left(\operatorname{Conv}\left(n_{s}, N_{1}\right)\right)$ for $N_{1}=300$. It can be seen that a reasonable convergence is reached for $n_{s}=300$. Fig. 4 displays the graph $N_{1} \mapsto$ $20 \log _{10}\left(\operatorname{Conv}\left(n_{s}, N_{1}\right)\right)$ for $n_{s}=300$. Convergence is reached for $N_{1}=225$.

\subsection{Target and its comparison with the reference observation.}

As explained above, the robust design optimization is carried out over the frequency band $\mathbb{B}_{1}=$ $[500,600] \mathrm{Hz}$. In band $\mathbb{B}_{1}$, there are three main resonances. The higher resonance occurs at $571 \mathrm{~Hz}$ with a level of $80.5 \mathrm{~dB}$ (see Fig. 5). There are two secondary resonances with a smaller level. The target is specified in order that the two secondary responses be not modified and that to reduce strongly the higher resonance. Figure 5 shows the target $\omega \mapsto 20 \log _{10}(g(\omega))$ related to the reference observation $\omega \mapsto 20 \log _{10}\left(\underline{w}^{\operatorname{master}}(\omega)\right)$ defined in Section 6.2. 


\subsection{Case 1 : robust design optimization with respect to a single design parameter.}

In the present case, the masses of the oscillators of each vibration absorber constituting the subsystem are assumed to be identical. The design parameter is the total mass $m$ of the subsystem. The robust design optimization is carried out with $\delta_{M}^{1}=\delta_{D}^{1}=\delta_{K}^{1}=0.05$ for the master system and with $\delta_{M}^{2}=\delta_{D}^{2}=\delta_{K}^{2}=0$ for the subsystem (no uncertainties in the subsystem). Note that the subsystem is assumed to be deterministic for convenience, the generalization to uncertain subsystems being straightforward. It has been verified that the numerical parameters identified from the convergence analysis of the stochastic master system also yield convergent results for the stochastic reduced system. Consequently, the robust design optimization is carried out with $N_{1}=225, N_{2}=54$ and $n_{s}=300$. The admissible set for design parameter $\mathrm{m}$ is defined such that $\mathrm{m} \in\left[4.5 \times 10^{-7}, 1.8 \times 10^{-3}\right] \mathrm{Kg}$. We are interested in comparing the design optimization (no uncertainties) with the robust design optimization (with uncertainties) for a weighting factor $\alpha$ which is chosen as 0.5 . The design optimization yields optimal design parameters $\mathrm{m}^{\mathrm{D}}=2.6 \times 10^{-4} \mathrm{Kg}$ and $\mathrm{m}^{\mathrm{RD}}=8.12 \times 10^{-4} \mathrm{Kg}$. A stochastic dynamical analysis of each one of the two optimal designs is then carried out in order to analyze the sensitivity of these two optimal designs with respect to model and data uncertainties. Let $\mu^{\mathrm{D}}(\omega)$, $\mu^{\mathrm{RD}}(\omega)$ and $\sigma^{\mathrm{D}}(\omega), \sigma^{\mathrm{RD}}(\omega)$ be the mean values and the standard deviations of the random observations $W^{\mathrm{D}}(\omega)$ and $W^{\mathrm{RD}}(\omega)$ defined by $W^{\mathrm{D}}(\omega)=W\left(\mathrm{~m}^{\mathrm{D}}, \omega\right), W^{\mathrm{RD}}(\omega)=W\left(\mathrm{~m}^{\mathrm{RD}}, \omega\right)$. We obtain $j\left(\mathrm{~m}^{\mathrm{D}}, \frac{1}{2}\right)=7.9835 \times 10^{-3}$ with $\left\|\sigma^{\mathrm{D}}\right\|_{\mathbb{B}_{1}}=6.1924 \times 10^{-2}\|g\|_{\mathbb{B}_{1}}$ and $j\left(\mathrm{~m}^{\mathrm{RD}}, \frac{1}{2}\right)=$ $7.2899 \times 10^{-3}$ with $\left\|\sigma^{\mathrm{RD}}\right\|_{\mathbb{B}_{1}}=5.8034 \times 10^{-2}\|g\|_{\mathbb{B}_{1}}$. Clearly, the robust design optimization yields the optimal design point whether it is with respect to the target performance objective or with respect to the robustness performance objective.

Below, the sensitivity of the robust design optimization is analyzed with respect to the weighting factor $\alpha \in] 0,1 / 2]$. From Fig. 6, it can be seen that the mass of the robust design decreases with the weighting factor $\alpha$. For this application, the sensitivities of the cost function and of the normalized standard deviation $\frac{\left\|\sigma^{\mathrm{RD}}\right\|_{\mathbb{B}_{1}}}{\left\|\sigma^{\mathrm{D}}\right\|_{\mathrm{B}_{1}}}$ are sufficiently weak with respect to the weighting factor (lower than $1.5 \%$ ) to be neglected. For this reason, all the results concerning the robust design optimization are presented for $\alpha=1 / 2$. 
Similarly to the stochastic case, let $\underline{w}^{\mathrm{D}}(\omega)=\underline{w}\left(\mathrm{~m}^{\mathrm{D}}, \omega\right)$ and $\underline{w}^{\mathrm{RD}}(\omega)=\underline{w}\left(\mathrm{~m}^{\mathrm{RD}}, \omega\right)$. Figure 7 displays the comparison of the target with the response of the mean models corresponding to the design optimization point and corresponding to the robust design optimization point. It can be seen from this Figure that the responses of the mean model corresponding to these two design points are different. In particular, the deterministic design point yields a response of the mean model which is closer to the target than to the robust design point. Nevertheless, this kind of information is not essential (see below). For all $\omega$ fixed in $\mathbb{B}$, the confidence interval of the random variable $W^{\mathrm{RD}}(\omega)$ is constructed for a probability level $P_{c}=0.95$ using the sample quantiles [37]. Let $\widetilde{W}^{\mathrm{RD}}\left(\theta_{1}, \omega\right) \leq \ldots \leq \widetilde{W}^{\mathrm{RD}}\left(\theta_{n_{s}}, \omega\right)$ be the order statistics. The upper interval $w^{+}(\omega)$ and the lower interval $w^{-}(\omega)$ delimiting the confidence interval with probability level $P_{c}$ is then given by

$$
\begin{aligned}
& w^{+}(\omega)=\tilde{W}^{\mathrm{RD}}\left(\theta_{k_{+}}, \omega\right) \quad, \quad k_{+}=\operatorname{fix}\left(0.5 n_{s}\left(1+P_{c}\right)\right) \\
& w^{-}(\omega)=\tilde{W}^{\mathrm{RD}}\left(\theta_{k_{-}}, \omega\right) \quad, \quad k_{-}=\operatorname{fix}\left(0.5 n_{s}\left(1-P_{c}\right)\right)
\end{aligned}
$$

in which fix $(x)$ is the integer part of real $x$. Fig. 8 compares the reference solution (response of the mean master system $) \omega \mapsto 20 \log _{10}\left(\underline{w}^{\text {master }}(\omega)\right)$ with the confidence region of the robust design optimization. In particular, the resonance of the reference solution occuring at frequency $571 \mathrm{~Hz}$ has been reduced (about of $3 \mathrm{~dB}$ or $4 \mathrm{~dB}$ ) by the robust design optimization process. It can be seen that the response of the mean master system belongs to the confidence region of the response of the stochastic system corresponding to the robust design optimization except in the frequency band $\mathbb{B}_{1}$ for which the target is active. Figure 9 and Fig. 10 compare the reference solution $\omega \mapsto 20 \log _{10}\left(\underline{w}^{\text {master }}(\omega)\right)$ with the confidence regions of the random responses $\omega \mapsto 20 \log _{10}\left(W^{\mathrm{D}}(\omega)\right)$ corresponding to the design optimization and $\omega \mapsto 20 \log _{10}\left(W^{\mathrm{RD}}(\omega)\right)$ corresponding to the robust design optimization for a probability level $P_{c}=0.95$ in the frequency band $\mathbb{B}_{1}$. In Fig. 10, there are five resonances which occur at frequencies $508 \mathrm{~Hz}, 524 \mathrm{~Hz}$, $539 \mathrm{~Hz}, 571 \mathrm{~Hz}$ and $583 \mathrm{~Hz}$. In Fig. 10, for peaks number 1 and 4, it can be seen that the robust design optimization yields similar results to the design optimization. For peaks number 2 and 5 , the robust design optimization yields lower responses levels. Moreover, the confidence region is particularly narrow in the frequency band $[550,600] \mathrm{Hz}$ which means that the optimum design is more robust with respect to model and data uncertainties than in the frequency band 
$[500,550] \mathrm{Hz}$. Finally, a sensitivity analysis with respect to the level of uncertainty in the dynamical system is carried out. Figure 11 displays the graph of $\boldsymbol{\delta} \mapsto \mathrm{m}^{\mathrm{RD}}(\boldsymbol{\delta})$ for $\alpha=0.1$ (marker ○), for $\alpha=0.25$ (marker $\diamond$ ) and for $\alpha=0.5$ marker $\square$ ).

\subsection{Case 2 : robust design optimization with respect to a multi-dimensional design parameter.}

In this subsection, no assumption is made on the mass of the oscillators of each vibration absorbers. Denoting as $m_{i}$ the mass of the oscillator number $i$, the design parameter $\mathrm{m}$ is the $\mathbb{R}^{5}$,vector $\mathfrak{m}=\left(9 m_{1}, 9 m_{2}, 9 m_{3}, 9 m_{4}, 9 m_{5}\right)$. The admissible set for design parameter $\mathrm{m}$ is such that $m$ belongs to the five dimensional hypercube defined by $m \in\left[9 \times 10^{-8}, 3.6 \times 10^{-4}\right]^{5}$. First, the design optimization (no uncertainties) is carried out for several initial values of m which are randomly chosen in order to obtain the best local optimum. The design optimization yields an optimal design parameter $\mathrm{m}^{D}=(4995,9,9,9,3024) \times 10^{-8}$. This optimal design parameter is then used as an initial value for the robust design optimization problem. The weighting factor $\alpha$ is set to $\alpha=0.5$. Two subcases for which the subsystem is deterministic are presented, (1) a low level of uncertainty in the master system $\left(\delta_{M}^{1}=\delta_{D}^{1}=\delta_{K}^{1}=0.05\right)$ yielding $\mathrm{m}^{\mathrm{RD}}=(6894,9,9,9,3582) \times 10^{-8}$, (2) a medium level of uncertainty in the master system $\left(\delta_{M}^{1}=\delta_{D}^{1}=\delta_{K}^{1}=0.15\right)$ yielding $\mathrm{m}^{\mathrm{RD}}=(1422,9,9,9,1062) \times 10^{-8}$. For each case, Figure 12 and 13 compare the reference solution $\omega \mapsto 20 \log _{10}\left(\underline{w}^{\text {master }}(\omega)\right)$ with the $10,20,30,40,50,60,70,80,90$ th quantiles of the random responses $\omega \mapsto 20 \log _{10}\left(W^{\mathrm{D}}(\omega)\right)$ corresponding to the design optimization and of the random responses $\omega \mapsto 20 \log _{10}\left(W^{\mathrm{RD}}(\omega)\right)$ corresponding to the robust design optimization in the frequency band $\mathbb{B}_{1}$. For the low uncertainty subcase, it can be seen in Fig. 12 that robust design optimization yields similar results to design optimization except for peak number 4 for which the response level is lower that corresponds to the target specification. For the medium uncertainty subcase, Figure 13 shows that robust design optimization yields similar results to design optimization except for peak number 4 for which the response level is lower and for peak 5 for which the response level is higher. In this case, the difference between the two cost functions is very small which means that, for the dynamical system under consideration, the robust design optimization does not improve the design optimization for a higher level of uncertainty. Note that design optimization shiftes the 
position of peak number 4 and 5 to the left and to the right respectively. Finally, it should be noted that both design optimization and robust design optimization have been solved by using a sequential quadratic optimization algorithm. The calculations have been made (1) in supplying the gradients of the cost function to the algorithm and (2) in supplying the gradient and the Hessian of the cost function to the algorithm using the numerical method proposed in the Appendix. Method (2) allows a CPU time gain about $30 \%$ to be obtained.

\section{Conclusion}

In this paper, we have presented an approach which allows the robust design optimization problem to be formulated and solved in presence of model uncertainties. Model uncertainties are taken into account with a nonparametric probabilistic approach and a target is introduced in the cost function. Thanks to an adapted algebraic development and under several assumptions, the numerical optimization problem can be solved with accuracy and with a low numerical cost. The proposed robust design formulation is general enough and the proposed approach can be used in other cases for analyzing complex dynamical systems in computational mechanics. Concerning the limitations of the proposed method, it should be noted that the gradient and the Hessian algebraically calculated can be used if the dynamical stiffness operator and the input force are affine mappings of the vector-valued design parameter and if the projection basis can be constructed independently of the vector-valued design parameter and has reasonable dimension. Not that if these conditions are not satisfied, the theory presented can be used with an optimization algorithm for which either the gradient and Hessian are not given or the gradient and Hessian are numerically calculated. The case of random loads is not investigated in this work but the extension is straightforward. In addition, this paper is focused on robust design with respect to model uncertainties. With respect to potential difficulties induced by the optimization algorithm when the dimension of the vector-valued design parameter is high, the proposed method does not introduce new difficulties with respect to the state of the art. The method has been validated for the five-dimensional case. As a final comment, the two numerical examples presented in this paper show that there are differences between design optimization and robust design optimization for the structural dynamics problem considered. 


\section{Acknowledgements}

The French National Research Agency (ANR) is acknowledged for supporting this research in the context of the CORODYNA project (Ref. ANR-05-BLAN-0082-01). 


\section{A Numerical analysis related to the optimization}

In this appendix, we summarize the algebraic development allowing the gradient and the Hessian of cost function $j(\mathbf{p}, \alpha)$ related to the robust design optimization problem to be computed. Such an algebraic development allows the precision in the optimization algorithm to be increased. The developments presented in this appendix are valuable under the following assumptions: (1) the dynamic stiffness operator and the force vector are affine functions of the design parameter, (2) the projection basis related to the dynamical substructuring reduction method does not depend of the design parameter.

\section{A.1 Notation}

Let $[A]$ and $[B]$ be $m \times n$ and $p \times q$ complex matrices. The operator $\star$ is introduced such that $[A] \star[B]$ is the $m p \times n q$ complex matrix defined by

$$
[A] \star[B]=\left[\begin{array}{ccc}
{[A]_{11}[B]} & \ldots & {[A]_{1 n}[B]} \\
\vdots & \ddots & \vdots \\
{[A]_{m 1}[B]} & \ldots & {[A]_{m n}[B]}
\end{array}\right] .
$$

Let $\mathbf{b}(\mathbf{p})$ be a vector in $\mathbb{C}^{n}$ and let $[A(\mathbf{p})]$ be a $m \times n$ complex matrix which depends on the vector $\mathbf{p}=\left(p_{1}, \ldots, p_{s}\right)$ in $\mathbb{C}^{s}$. Below, when no confusion is possible, the argument $\mathbf{p}$ is removed in order to simplify the writing.

The vector $\mathscr{I}_{\mathbf{b}}(\mathbf{p})$ is the vector in $\mathbb{C}^{n s}$ defined by $\mathfrak{g}_{\mathbf{b}}(\mathbf{p})=\left\{\partial_{p_{1}} \mathbf{b}, \ldots, \partial_{p_{s}} \mathbf{b}\right\}$.

The vector $\mathfrak{h}_{\mathbf{b}}(\mathbf{p})$ is the vector in $\mathbb{C}^{n s(s+1) / 2}$ defined by

$\bigcap_{\mathbf{b}}(\mathbf{p})=\left\{\partial_{p_{1}^{2}}^{2} \mathbf{b}, \ldots, \partial_{p_{1} p_{s}}^{2} \mathbf{b}, \partial_{p_{2}^{2}}^{2} \mathbf{b}, \ldots, \partial_{p_{2} p_{s}}^{2} \mathbf{b}, \ldots, \partial_{p_{s}^{2}}^{2} \mathbf{b}\right\}$

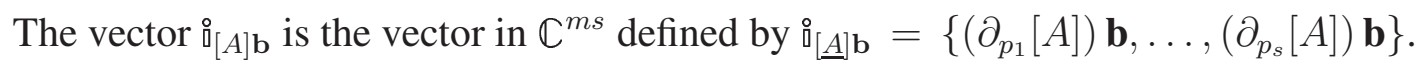

The vector $\AA_{[A] \mathbf{b}}(\mathbf{p})$ is the vector in $\mathbb{C}^{m s(s+1) / 2}$ defined by 
$\stackrel{\bigotimes}{[}[A] \mathbf{b}=\left\{\left(\partial_{p_{1}}[A]\right) \partial_{p_{1}} \mathbf{b}, \ldots,\left(\partial_{p_{1}}[A]\right) \partial_{p_{s}} \mathbf{b},\left(\partial_{p_{2}}[A]\right) \partial_{p_{2}} \mathbf{b}, \ldots,\left(\partial_{p_{2}}[A]\right) \partial_{p_{s}} \mathbf{b}, \ldots,\left(\partial_{p_{s}}[A]\right) \partial_{p_{s}} \mathbf{b}\right\}$.

The vector $\mathbb{k}_{[A] \mathrm{b}}(\mathbf{p})$ is the vector in $\mathbb{C}^{m s(s+1) / 2}$ defined by

$\mathbb{k}_{[A] \mathbf{b}}=\left\{\left(\partial_{p_{1}^{2}}^{2}[A]\right) \mathbf{b}, \ldots,\left(\partial_{p_{1} p_{s}}^{2}[A]\right) \mathbf{b},\left(\partial_{p_{2}^{2}}^{2}[A]\right) \mathbf{b}, \ldots,\left(\partial_{p_{2} p_{s}}^{2}[A]\right) \mathbf{b}, \ldots,\left(\partial_{p_{s}}^{2}[A]\right) \mathbf{b}\right\}$

A.2 Gradient and Hessian of the cost function for the stochastic dynamical system

By using Eqs. (20), the first-order and the second-order partial derivatives of the cost function $j(\mathbf{p}, \alpha)$ with respect to $\mathbf{p}$ are written as

$$
\begin{aligned}
\partial_{p_{i}} j(\mathbf{p}, \alpha)= & \frac{1}{\|\mathbf{g}\|_{\mathbb{B}_{1}}} \mathcal{E}\left\{2 \mathcal{R} e \int_{\mathbb{B}_{1}} \alpha(\mathbf{W}(\mathbf{p}, \omega)-\mathbf{g}(\omega))^{*} \partial_{p_{i}} \mathbf{W}(\mathbf{p}, \omega)\right. \\
& \left.+(1-2 \alpha)(\mathbf{W}(\mathbf{p}, \omega)-\boldsymbol{\mu}(\mathbf{p}, \omega))^{*} \partial_{p_{i}}(\mathbf{W}(\mathbf{p}, \omega)-\boldsymbol{\mu}(\mathbf{p}, \omega)) d \omega\right\} \\
\partial_{p_{i} p_{j}}^{2} j(\mathbf{p}, \alpha)= & \frac{1}{\|\mathbf{g}\|_{\mathbb{B}_{1}}} \mathcal{E}\left\{2 \mathcal{R} e \int_{\mathbb{B}_{1}} \alpha(\mathbf{W}(\mathbf{p}, \omega)-\mathbf{g}(\omega))^{*} \partial_{p_{i} p_{j}}^{2} \mathbf{W}(\mathbf{p}, \omega)\right. \\
& +\alpha \partial_{p_{j}} \mathbf{W}(\mathbf{p}, \omega)^{*} \partial_{p_{i}} \mathbf{W}(\mathbf{p}, \omega) \\
& +(1-2 \alpha)(\mathbf{W}(\mathbf{p}, \omega)-\mathbf{g}(\omega))^{*} \partial_{p_{i} p_{j}}^{2}(\mathbf{W}(\mathbf{p}, \omega)-\boldsymbol{\mu}(\mathbf{p}, \omega)) \\
& \left.+(1-2 \alpha) \partial_{p_{j}}(\mathbf{W}(\mathbf{p}, \omega)-\mathbf{g}(\omega))^{*} \partial_{p_{i}}(\mathbf{W}(\mathbf{p}, \omega)-\boldsymbol{\mu}(\mathbf{p}, \omega)) d \omega\right\}
\end{aligned}
$$

with summation over indices $k$, in which $\mathcal{E}$ is the mathematical expectation, $\mathcal{R} e$ is the real part of a complex number and where a starred vector is its transconjuguate. By using Eqs. (14) and (18), it can easily be shown that the $\mathbb{C}^{k}$-valued random vectors $\mathbf{W}(\mathbf{p}, \omega), \partial_{p_{i}} \mathbf{W}(\mathbf{p}, \omega)$ for $i \in\{1, \ldots, s\}, \partial_{p_{i} p_{j}}^{2} \mathbf{W}(\mathbf{p}, \omega)$ for $1 \leq i \leq j \leq s$ can be calculated from the $\mathbb{C}^{N}$-valued random vectors $\mathbf{Q}(\mathbf{p}, \omega), \partial_{p_{i}} \mathbf{Q}(\mathbf{p}, \omega)$ for $i \in\{1, \ldots, s\}, \partial_{p_{i} p_{j}}^{2} \mathbf{Q}(\mathbf{p}, \omega)$ for $1 \leq i \leq j \leq s$. 


\section{A.3 Gradient and Hessian of the random vector of the random reduced coordinates}

Let $\mathcal{Q}(\mathbf{p}, \omega)$ be the $\mathbb{C}^{N S}$-valued random vector defined by

$$
\mathcal{Q}(\mathbf{p}, \omega)=\left[\begin{array}{c}
\mathbf{Q}(\mathbf{p}, \omega) \\
\mathfrak{I}_{\mathbf{Q}}(\mathbf{p}, \omega) \\
\mathfrak{h}_{\mathbf{Q}}(\mathbf{p}, \omega)
\end{array}\right],
$$

in which $S=1+3 s / 2+5 s^{2} / 2$ and $N$ is the dimension of vector $\mathbf{Q}(\mathbf{p}, \omega)$. Let $\left[\mathcal{A}_{\text {red }}(\mathbf{p}, \omega)\right]$ be the random matrix $\left[\mathcal{A}_{\text {red }}(\mathbf{p}, \omega)\right]$ with values in the set of all the symmetric $N S \times N S$ complex matrices and let $\mathcal{F}_{\text {red }}(\mathbf{p}, \omega)$ be the $\mathbb{C}^{N S}$-valued random vector such that

$$
\begin{aligned}
& {\left[\mathcal{A}_{\text {red }}(\mathbf{p}, \omega)\right]=\left[I_{S}\right] \star\left(\left[\mathbf{A}_{\text {red }}^{1}(\omega)\right]+\left[\mathbf{A}_{\text {red }}^{2}(\mathbf{p}, \omega)\right]\right)} \\
& \mathcal{F}_{\text {red }}(\mathbf{p}, \omega)=\left[\begin{array}{c}
\mathbf{f}_{\text {red }}(\mathbf{p}, \omega) \\
\mathbb{I}_{\mathrm{f}_{\text {red }}}(\mathbf{p}, \omega)-\stackrel{\circ}{[}_{\left[\mathbf{A}_{\text {red }}^{2}\right] \mathbf{Q}}(\mathbf{p}, \omega) \\
-\AA_{\left[\mathbf{A}_{\text {red }}^{2}\right] \mathbf{Q}}(\mathbf{p}, \omega)-\mathbb{k}_{\left[\mathbf{A}_{\text {red }}^{2}\right] \mathbf{Q}}(\mathbf{p}, \omega)
\end{array}\right]
\end{aligned}
$$

in which $\left[I_{S}\right]$ is the $S \times S$ identity matrix. From Eq. 15 and taking into account that $\left[\mathbf{A}_{\text {red }}^{1}(\omega)\right]$ is independent of $\mathbf{p}$, it can be shown that the random vector $\mathcal{Q}(\mathbf{p}, \omega)$ is solution of the random matrix equation

$$
\left[\mathcal{A}_{\text {red }}(\mathbf{p}, \omega)\right] \mathcal{Q}(\mathbf{p}, \omega)=\mathcal{F}_{\text {red }}(\mathbf{p}, \omega)
$$

It should be noted that the calculation of ${\stackrel{\circ}{[}\left[\mathbf{A}_{\text {red }}^{2}\right] \mathbf{Q}}(\mathbf{p}, \omega), \AA_{\left[\mathbf{A}_{\text {red }}^{2}\right] \mathbf{Q}}(\mathbf{p}, \omega)$ and $\mathbb{k}_{\left[\mathbf{A}_{\text {red }}^{2} \mathbf{Q}\right.}(\mathbf{p}, \omega)$ requires the calculation of the partial derivatives $\partial_{p_{i}}\left[\mathbf{A}_{\text {red }}^{2}(\mathbf{p}, \omega)\right]$ for $i=\{1, \ldots, s\}$ and $\partial_{p_{i} p_{j}}^{2}\left[\mathbf{A}_{\text {red }}^{2}(\mathbf{p}, \omega)\right]$ for $1 \leq i \leq j \leq s$. This calculation is carried out in the next Section.

\section{A.4 Gradient of the random reduced dynamical stiffness matrix for the stochastic dynamical} system

The method allowing the partial derivative $\partial_{p_{i}}\left[\mathbf{A}_{\text {red }}^{2}(\mathbf{p}, \omega)\right]$ to be algebraically calculated for $i=\{1, \ldots, s\}$ is presented below. From Eqs. (16) and (17), it can be seen that the calculation of $\partial_{p_{i}}\left[\mathbf{A}_{\text {red }}^{2}(\mathbf{p}, \omega)\right]$ requires the calculation of $\partial_{p_{i}}\left[\mathbf{M}_{s}^{2}(\mathbf{p})\right], \partial_{p_{i}}\left[\mathbf{D}_{s}^{2}(\mathbf{p})\right]$ and $\partial_{p_{i}}\left[\mathbf{K}_{s}^{2}(\mathbf{p})\right]$. Rewritting Eq. (12) for matrices $\left[\mathbf{M}_{s}^{2}(\mathbf{p})\right],\left[\mathbf{D}_{s}^{2}(\mathbf{p})\right]$ and $\left[\mathbf{K}_{s}^{2}(\mathbf{p})\right]$ yields 


$$
\left[\mathbf{E}_{s}^{2}(\mathbf{p})\right]=\left[\underline{L}_{E}^{2}(\mathbf{p})\right]^{T}\left[\mathbf{G}_{E}^{2}\right]\left[\underline{L}_{E}^{2}(\mathbf{p})\right],
$$

in which $\left[\mathbf{E}_{s}^{2}(\mathbf{p})\right]$ denotes $\left\{\left[\mathbf{M}_{s}^{2}(\mathbf{p})\right],\left[\mathbf{D}_{s}^{2}(\mathbf{p})\right],\left[\mathbf{K}_{s}^{2}(\mathbf{p})\right]\right\},\left[\underline{L}_{E}^{2}(\mathbf{p})\right]$ denotes $\left\{\left[\underline{L}_{M}^{2}(\mathbf{p})\right],\left[\underline{L}_{D}^{2}(\mathbf{p})\right],\left[\underline{L}_{K}^{2}(\mathbf{p})\right]\right\}$ and $\left[\mathbf{G}_{E}^{2}\right]$ denotes $\left\{\left[\mathbf{G}_{M}^{2}\right],\left[\mathbf{G}_{D}^{2}\right],\left[\mathbf{G}_{K}^{2}\right]\right\}$. In Eq. (A-8), the matrix $\left[\mathbf{G}_{M}^{2}\right]$ is a random matrix with values in the set of all the symmetric $N_{2} \times N_{2}$ positive definite matrices and matrices $\left[\mathbf{G}_{D}^{2}\right],\left[\mathbf{G}_{K}^{2}\right]$ are random matrices with values in the set of all the symmetric $\left(N_{2}-r\right) \times\left(N_{2}-r\right)$ positive definite matrices. Matrix $\left[\underline{L}_{E}^{2}(\mathbf{p})\right]$ is such that

$$
\left[\underline{E}_{s}^{2}(\mathbf{p})\right]=\left[\underline{L}_{E}^{2}(\mathbf{p})\right]^{T}\left[\underline{L}_{E}^{2}(\mathbf{p})\right]
$$

in which $\left[\underline{L}_{M}^{2}(\mathbf{p})\right]$ is a $N_{2} \times N_{2}$ upper triangular real matrix obtained by Cholesky factorization and where $\left[\underline{L}_{D}^{2}(\mathbf{p})\right],\left[\underline{L}_{K}^{2}(\mathbf{p})\right]$ are $N_{2} \times\left(N_{2}-r\right)$ real matrices. In addition, according to Eq. (3), the matrix $\left[\underline{E}_{s}^{2}(\mathbf{p})\right]$ can be written as

$$
\left[\underline{E}_{s}^{2}(\mathbf{p})\right]=\left[\underline{E}_{s}^{2,0}\right]+\sum_{i=1}^{s} p_{i}\left[\underline{E}_{s}^{2, i}\right]
$$

Taking the partial derivative of Eq. (A-8) with respect to $p_{i}$, the matrix $\partial_{p_{i}}\left[\mathbf{E}_{s}^{2}(\mathbf{p})\right]$ is given by

$$
\partial_{p_{i}}\left[\mathbf{E}_{s}^{2}(\mathbf{p})\right]=\left(\partial_{p_{i}}\left[\underline{L}_{E}^{2}(\mathbf{p})\right]^{T}\right)\left[\mathbf{G}_{E}^{2}\right]\left[\underline{L}_{E}^{2}(\mathbf{p})\right]+\left[\underline{L}_{E}^{2}(\mathbf{p})\right]^{T}\left[\mathbf{G}_{E}^{2}\right]\left(\partial_{p_{i}}\left[\underline{L}_{E}^{2}(\mathbf{p})\right]\right)
$$

Using Eq. (A-9) and (A-10), the matrix $\partial_{p_{i}}\left[\underline{L}_{E}^{2}(\mathbf{p})\right]$ is computed in solving the following matrix equation

$$
\left[\underline{E}_{s}^{2, i}\right]=\left(\partial_{p_{i}}\left[\underline{L}_{E}^{2}(\mathbf{p})\right]^{T}\right)\left[\underline{L}_{E}^{2}(\mathbf{p})\right]+\left[\underline{L}_{E}^{2}(\mathbf{p})\right]^{T}\left(\partial_{p_{i}}\left[\underline{L}_{E}^{2}(\mathbf{p})\right]\right)
$$

\section{A.5 Hessian of the random reduced dynamical stiffness matrix for the stochastic dynamical} system

The method allowing the partial derivative $\partial_{p_{i} p_{j}}^{2}\left[\mathbf{A}_{\text {red }}^{2}(\mathbf{p}, \omega)\right]$ to be algebraically calculated for $1 \leq i \leq j \leq s$ is presented. Taking the partial derivative of Eq. (A-11) with respect to $p_{j}$, the matrix $\partial_{p_{i} p_{j}}^{2}\left[\mathbf{E}_{s}^{2}(\mathbf{p})\right]$ is given by given by 


$$
\begin{aligned}
& \partial_{p_{i} p_{j}}^{2}\left[\mathbf{E}_{s}^{2}(\mathbf{p})\right]=\left(\partial_{p_{i} p_{j}}^{2}\left[\underline{L}_{E}^{2}(\mathbf{p})\right]^{T}\right)\left[\mathbf{G}_{E}^{2}\right]\left[\underline{L}_{E}^{2}(\mathbf{p})\right]+\left(\partial_{p_{i}}\left[\underline{L}_{E}^{2}(\mathbf{p})\right]^{T}\right)\left[\mathbf{G}_{E}^{2}\right]\left(\partial_{p_{j}}\left[\underline{L}_{E}^{2}(\mathbf{p})\right]\right)(\mathrm{A}-13) \\
& +\left(\partial_{p_{j}}\left[\underline{L}_{E}^{2}(\mathbf{p})\right]^{T}\right)\left[\mathbf{G}_{E}^{2}\right]\left(\partial_{p_{i}}\left[\underline{L}_{E}^{2}(\mathbf{p})\right]\right)+\left[\underline{L}_{E}^{2}(\mathbf{p})\right]^{T}\left[\mathbf{G}_{M}^{2}\right]\left(\partial_{p_{i} p_{j}}^{2}\left[\underline{L}_{E}^{2}(\mathbf{p})\right]\right)
\end{aligned}
$$

Taking the partial derivative of Eq. (A-12), the matrix $\partial_{p_{i} p_{j}}^{2}\left[\underline{L}_{E}^{2}(\mathbf{p})\right]$ is computed by solving the following matrix equation

$$
\begin{array}{r}
-\left(\partial_{p_{i}}\left[\underline{L}_{E}^{2}(\mathbf{p})\right]^{T}\right)\left(\partial_{p_{j}}\left[\underline{L}_{E}^{2}(\mathbf{p})\right]\right)-\left(\partial_{p_{j}}\left[\underline{L}_{E}^{2}(\mathbf{p})\right]^{T}\right)\left(\partial_{p_{i}}\left[\underline{L}_{E}^{2}(\mathbf{p})\right]\right)= \\
\left(\partial_{p_{i} p_{j}}^{2}\left[\underline{L}_{E}^{2}(\mathbf{p})\right]^{T}\right)\left[\underline{L}_{E}^{2}(\mathbf{p})\right]+\left[\underline{L}_{E}^{2}(\mathbf{p})\right]^{T}\left(\partial_{p_{i} p_{j}}^{2}\left[\underline{L}_{E}^{2}(\mathbf{p})\right]\right) .
\end{array}
$$




\section{References}

[1] Petiau, C., 1991. "Structural optimization of aircraft". Thin-Walled Structures, 11 (1-2) , pp. 43-64.

[2] Ghanem, R., and Spanos, P., 1991. Stochastic finite elements:a spectral approach. Springer Verlag, New-York.

[3] Kleiber, M., Tran, D., and Hien, T., 1992. The stochastic finite element method. John Wiley and Sons, New-York.

[4] Ghanem, R., 1999. "Ingredients for a general purpose stochastic finite elements formulation". Computer Methods in Applied Mechanics and Engineering, 168 (1-4) , pp. 19-34.

[5] Babuska, I., and Chatzipantelidis, P., 2002. "On solving elliptic stochastic partial differential equations". Computer Methods in Applied Mechanics and Engineering, 191 (37-38) , pp. 40934122.

[6] Schuëller (Editor), G., 1997. "A state-of-the-art report on computational stochastic mechanics". Probabilistic Engineering Mechanics, 12 (4) , pp. 197-321.

[7] Pradlwarter, H., Schueller, G., and Szekely, G., 2002. "Random eigenvalue problems for large systems". Computers \& Structures, 80 (27-30) , pp. 2415-2424.

[8] Soize, C., 2005. "A comprehensive overview of a non-parametric probabilistic approach of model uncertainties for predictive models in structural dynamics". Journal of Sound and Vibration, 288 (3) , pp. 623-652.

[9] Soize, C., 2000. "A nonparametric model of random uncertainties for reduced matrix models in structural dynamics". Probabilistic Engineering Mechanics, 15 (3) , pp. 277-294.

[10] Soize, C., 2001. "Maximum entropy approach for modeling random uncertainties in transient elastodynamics.”. Journal of the Acoustical Society of America, 109 (5) , pp. 1979-1996.

[11] Soize, C., 2005. "Random matrix theory for modeling random uncertainties in computational mechanics". Computer Methods in Applied Mechanics and Engineering, 194 (12-16) , pp. 13331366.

[12] Taguchi, G., Elsayed, E., and Hsiang, T., 1989. Quality engineering in production systems. McGraw-Hill. 
[13] Parkinson, A., Sorensen, C., and Pouhassan, N., 1993. "A general approach for robust optimal design”. ASME Journal of Mechanical Design, 115 (1) , pp. 74-80.

[14] Ramakrishnan, B., and Rao, S., 1996. "A general loss function based optimization procedure for robust design". Engineering Optimization, 25 (4) , pp. 255-276.

[15] Lee, K.-H., and Park, G.-J., 2001. "Robust optimization considering tolerances of design variables". Computers \& Structures, 79 (1), pp. 77-86.

[16] Jung, D., and Lee, B., 2002. "Development of a simple and efficient method for robust design optimization”. International Journal for Numerical Methods in Engineering, 53 (9) , pp. 2201-2215.

[17] Sandgren, E., and Cameron, T., 2002. "Robust design optimization of structures through consideration of variation”. Computers \& Structures, 80 (20-21) , pp. 1605-1613.

[18] Doltsinis, I., and Kang, Z., 2004. "Robust design of structures using optimization methods". Computer Methods in Applied Mechanics and Engineering, 193 (23-26) , pp. 2221-2237.

[19] Zang, C., Friswell, M., and Mottershead, J., 2005. "A review of robust optimal design and its application in dynamics". Computers \& Structures, 83 (4-5) , pp. 315-326.

[20] Papadrakakis, M., Lagaros, N., and Plevris, V., 2005. "Design optimization of steel structures considering uncertainties”. Engineering Structures, 27 (9) , pp. 1408-1418.

[21] Duchereau, J., and Soize, C., 2006. "Transient dynamics in structures with nonhomogeneous uncertainties induced by complex joints". Mechanical Systems and Signal Processing, 20 (4) , pp. 954-967.

[22] Chebli, H., and Soize, C., 2004. "Experimental validation of a nonparametric probabilistic model of nonhomogeneous uncertainties for dynamical systems". Journal of the Acoustical Society of America, 115 (2) , pp. 697-705.

[23] Durand, J.-F., Gagliardini, L., and Soize, C., 2005. "Nonparametric modeling of the variability of vehicle vibroacoustic behavior". In Proceedings on the SAE Noise and Vibration Conference and Exhibition, Traverse City, Michigan, USA, 16-19 May 2005, ISBN 0768016576.

[24] Chen, C., Duhamel, D., and Soize, C., 2006. "Probabilistic approach for model and data uncertainties and its experimental identification in structural dynamics: case of composite sandwich panels". Journal of Sound and Vibration, 294 (1-2) , pp. 64-81. 
[25] Soize, C., 2005. "Probabilistic models for computational stochastic mechanics and applications". In Proceedings on the 9th International Conference on Structural Safety and Reliability ICOSSAR'05, Rome, Italy, 19-23 June 2005, G. Augusti, G.I. Schueller and M. Ciampoli, Millpress, Rotterdam, Netherlands, ISBN 9059660404.

[26] Capiez-Lernout, E., Soize, C., Lombard, J.-P., Dupont, C., and Seinturier, E., 2005. "Blade manufacturing tolerances definition for a mistuned industrial bladed disk". ASME Journal of Engineering for Gas Turbines and Power, 127 (3) , pp. 621-628.

[27] Capiez-Lernout, E., Pellissetti, M., Pradlwarter, H., Schueller, G., and Soize, C., 2006. "Data and model uncertainties in complex aerospace engineering systems". Journal of Sound and Vibration, 295 (3-5) , pp. 923-938.

[28] Craig, R., and Bampton, M., 1968. "Coupling of substructures for dynamic analyses". AIAA Journal, 6 (7) , pp. 1313-1319.

[29] Mac Neal, R., 1971. "A hybrid method of component mode synthesis". Computers \& Structures, 1 (4) , pp. 581-601.

[30] Benfield, W., and Hruda, R., 1971. "Vibration analysis of structures by component mode substitution". AIAA Journal, 9 (7), pp. 1255-1261.

[31] Rubin, S., 1975. "Improved component-mode representation for structural dynamic analysis". AIAA Journal, 13 (8) , pp. 995-1006.

[32] Morand, H.-P., and Ohayon, R., 1979. "Substructure variational analysis of the vibrations of coupled fluid-structure systems. finite element results.”. International Journal for Numerical Methods in Engineering, 14 (5) , pp. 741-755.

[33] Farhat, C., and Geradin, M., 1994. "On a component mode synthesis method and its application to incompatible substructures". Computers \& Structures, 51 (5) , pp. 459-473.

[34] Park, K.-C., 2004. "Partitioned component mode synthesis via a flexibility approach". AIAA Journal, 42 (6) , pp. 1236-1245.

[35] Fletcher, R., 1980. Practical methods of optimization,constrained optimization (Vol.2). John Wiley and Sons.

[36] Powell, M., 1983. "Variable metric methods for constrained optimization". Mathematical Programming: the state of the art, pp. 288-311. 
[37] Serfling, R., 1980. Approximation theorems of mathematical statistics. Wiley, New York. 
Figure 1: Finite element mesh of the dynamical system: attached spring (ם), attached lumped mass $(\bullet)$, attached set of 3 single DOF linear oscillators $(\triangle)$, vibration absorbers $(\boldsymbol{\Lambda})$, excitation node ( $)$, simply supported boundary (thick line), free boundary (thick dashed line).

Figure 2: Reference observation of the mean master system. Graph of function $\nu \mapsto 20 \log _{10}\left(\underline{w}^{\operatorname{master}}(2 \pi \nu)\right)$. Horizontal axis is the frequency $\nu$ in $\mathrm{Hz}$.

Figure 3: Convergence analysis : Graph of function $n_{s} \mapsto 20 \log _{10}\left(\operatorname{Conv}\left(n_{s}, N_{1}\right)\right)$ for the stochastic master system with $N_{1}=300$. Horizontal axis is $n_{s}$.

Figure 4: Convergence analysis : Graph of function $N_{1} \mapsto 20 \log _{10}\left(\operatorname{Conv}\left(n_{s}, N_{1}\right)\right)$ for the stochastic master system with $n_{s}=300$. Horizontal axis is $N_{1}$.

Figure 5: Definition of the target $\nu \mapsto 20 \log _{10}(g(2 \pi \nu))$ (thick dashed line). Comparison with the reference observation $\nu \mapsto 20 \log _{10}\left(\underline{w}^{\operatorname{master}}(2 \pi \nu)\right)$ (thin solid line) in the frequency band $\mathbb{B}_{1}=[500,600] \mathrm{Hz}$ (horizontal axis).

Figure 6: Case 1. Sensitivity of the robust design optimization with respect to the weighting factor $\alpha \in] 0,0.5]$ : Graph of $\alpha \mapsto \frac{\mathrm{m}^{\mathrm{RD}}}{\mathrm{m}^{\mathrm{D}}}$ (thick line), graph of $\alpha \mapsto j\left(\mathrm{~m}^{\mathrm{RD}}, \alpha\right)$ (thick dashed line), graph of $\alpha \mapsto \frac{\left\|\sigma^{\mathrm{RD}}\right\|_{\mathbb{B}_{1}}}{\left\|\sigma^{\mathrm{D}}\right\|_{\mathbb{B}_{1}}}$ (thin dashed line). Horizontal axis is $\alpha$.

Figure 7: Case 1. Comparison of the target $\nu \mapsto 20 \log _{10}(g(2 \pi \nu))$ (thick dashed line) with the response of the mean model corresponding to the design optimization $\nu \mapsto 20 \log _{10}\left(\underline{w}^{\mathrm{D}}(2 \pi \nu)\right)$ (thin dark gray line) and corresponding to the robust design optimization $\nu \mapsto 20 \log _{10}\left(\underline{w}^{\mathrm{RD}}(2 \pi \nu)\right)$ (thin light gray line) for $\alpha=1 / 2$. Horizontal axis is the frequency $\nu$ in $\mathrm{Hz}$.

Figure 8: Case 1. Comparison of the reference observation $\nu \mapsto 20 \log _{10}\left(\underline{w}^{\operatorname{master}}(2 \pi \nu)\right)$ (thin solid line) with the confidence region (light gray region) of random response for the robust 
design, over the band $\mathbb{B}=[5,1200] \mathrm{Hz}$ (horizontal axis) and for $\alpha=1 / 2$ and a probability level $P_{c}=0.95$. Horizontal axis is the frequency $\nu$ in $\mathrm{Hz}$.

Figure 9: Case 1. Comparison of the reference solution $\nu \mapsto 20 \log _{10}\left(\underline{w}^{\text {master }}(2 \pi \nu)\right)$ (thin solid line) with the confidence region (dark gray region) of the random response $\nu \mapsto 20 \log _{10}\left(W^{\mathrm{D}}(2 \pi \nu)\right)$ corresponding to the design optimization and with the confidence region (light gray region) of the random response $\nu \mapsto 20 \log _{10}\left(W^{\mathrm{RD}}(2 \pi \nu)\right)$ corresponding to the robust design optimization. Horizontal axis is the frequency $\nu$ in $\mathrm{Hz}$.

Figure 10: Case 1. Comparison of the reference solution $\nu \mapsto 20 \log _{10}\left(\underline{w}^{\operatorname{master}}(2 \pi \nu)\right)$ (thin solid line) with the two confidence regions defined in Fig. 9.

Figure 11: Case 1. Sensitivity analysis for the robust design optimization : Graph of function $\boldsymbol{\delta} \mapsto \mathrm{m}^{\mathrm{RD}}(\boldsymbol{\delta})$ for $\alpha=0.5$ (thin line with $\square$ ), for $\alpha=0.25$ (thin line with $\diamond$ ), for $\alpha=0.1$ (thin line with $\circ$ ). Horizontal axis is $\boldsymbol{\delta}$.

Figure 12: Case 2, low uncertainty level. Comparison of the reference solution $\nu \mapsto 20 \log _{10}\left(\underline{w}^{\operatorname{master}}(2 \pi \nu)\right)$ (thick solid line) with the 10, 20, 30, 40, 50, 60, 70, 80, 90th quantiles of the random response $\nu \mapsto 20 \log _{10}\left(W^{\mathrm{D}}(2 \pi \nu)\right)$ (thin black lines) corresponding to the design optimization and of the random response $\nu \mapsto 20 \log _{10}\left(W^{\mathrm{RD}}(2 \pi \nu)\right)$ (thin gray lines) corresponding to the robust design optimization. Horizontal axis is the frequency $\nu$ in $\mathrm{Hz}$.

Figure 13: Case 2, medium uncertainty level. Comparison of the reference solution $\nu \mapsto 20 \log _{10}\left(\underline{w}^{\operatorname{master}}(2 \pi \nu)\right)$ (thick solid line) with the 10, 20, 30, 40, 50, 60, 70, 80, 90th quantiles of the random response $\nu \mapsto 20 \log _{10}\left(W^{\mathrm{D}}(2 \pi \nu)\right)$ (thin black lines) corresponding to the design optimization and of the random response $\nu \mapsto 20 \log _{10}\left(W^{\mathrm{RD}}(2 \pi \nu)\right)$ (thin gray lines) corresponding to the robust design optimization. Horizontal axis is the frequency $\nu$ in $\mathrm{Hz}$. 


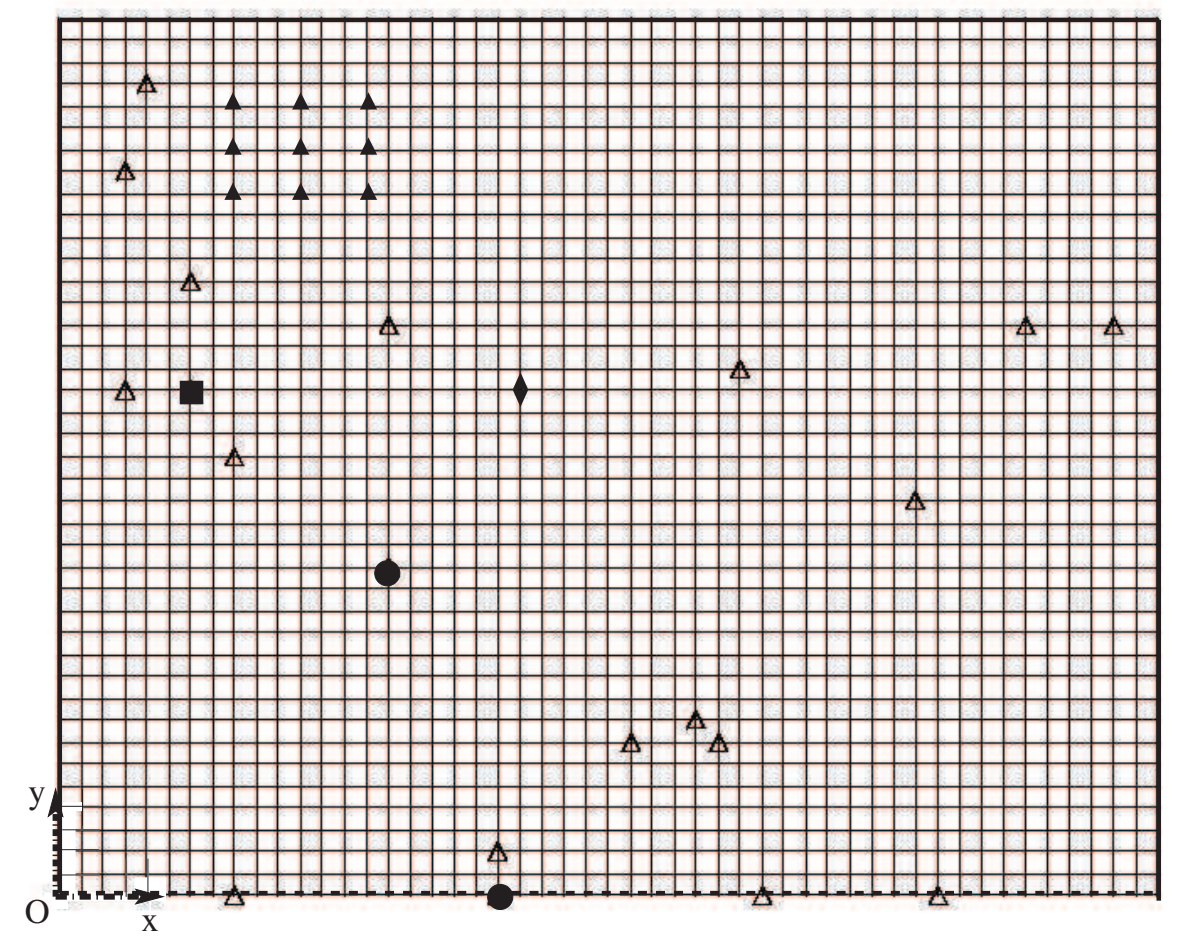

Fig. 1. Finite element mesh of the dynamical system: attached spring (ם), attached lumped mass $(\bullet)$, attached set of 3 single DOF linear oscillators $(\triangle)$, vibration absorbers $(\boldsymbol{\Delta})$, excitation node $(\checkmark)$, simply supported boundary (thick line), free boundary (thick dashed line) 


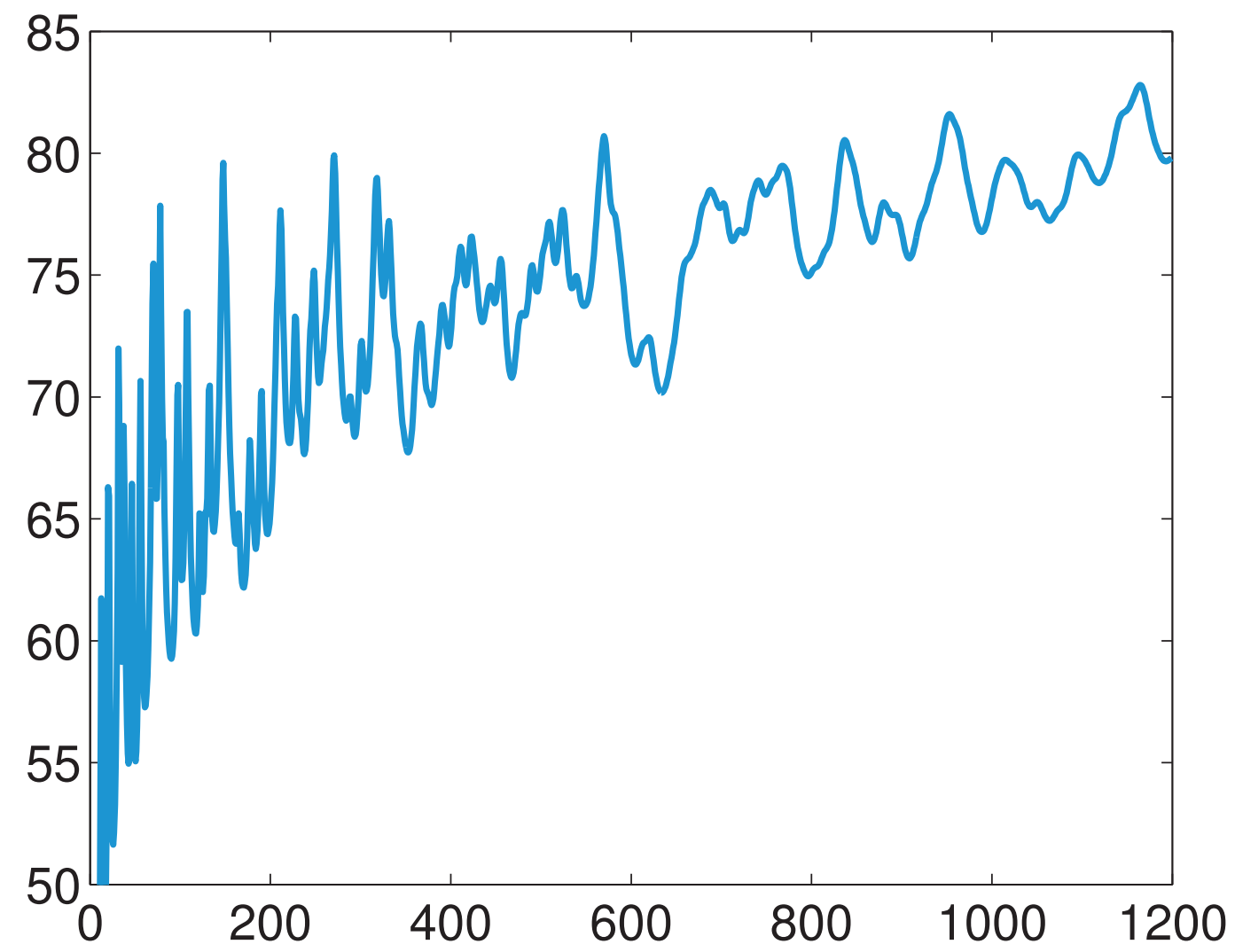

Fig. 2. Reference observation of the mean master system. Graph of function $\nu \mapsto 20 \log _{10}\left(\underline{w}^{\operatorname{master}}(2 \pi \nu)\right)$. Horizontal axis is the frequency $\nu$ in $\mathrm{Hz}$. 


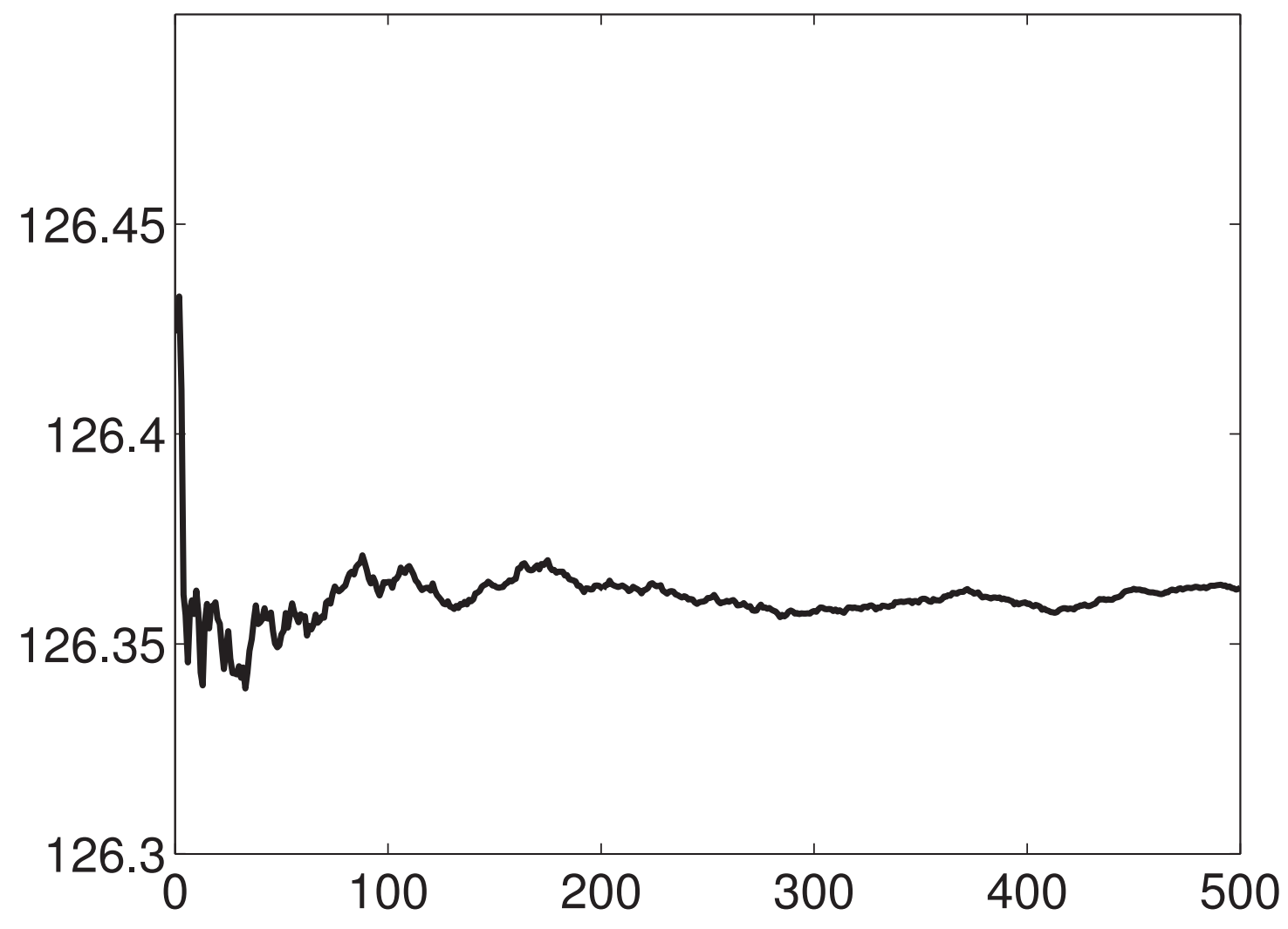

Fig. 3. Convergence analysis : Graph of function $n_{s} \mapsto 20 \log _{10}\left(\operatorname{Conv}\left(n_{s}, N_{1}\right)\right)$ for the stochastic master system with $N_{1}=300$. Horizontal axis is $n_{s}$. 


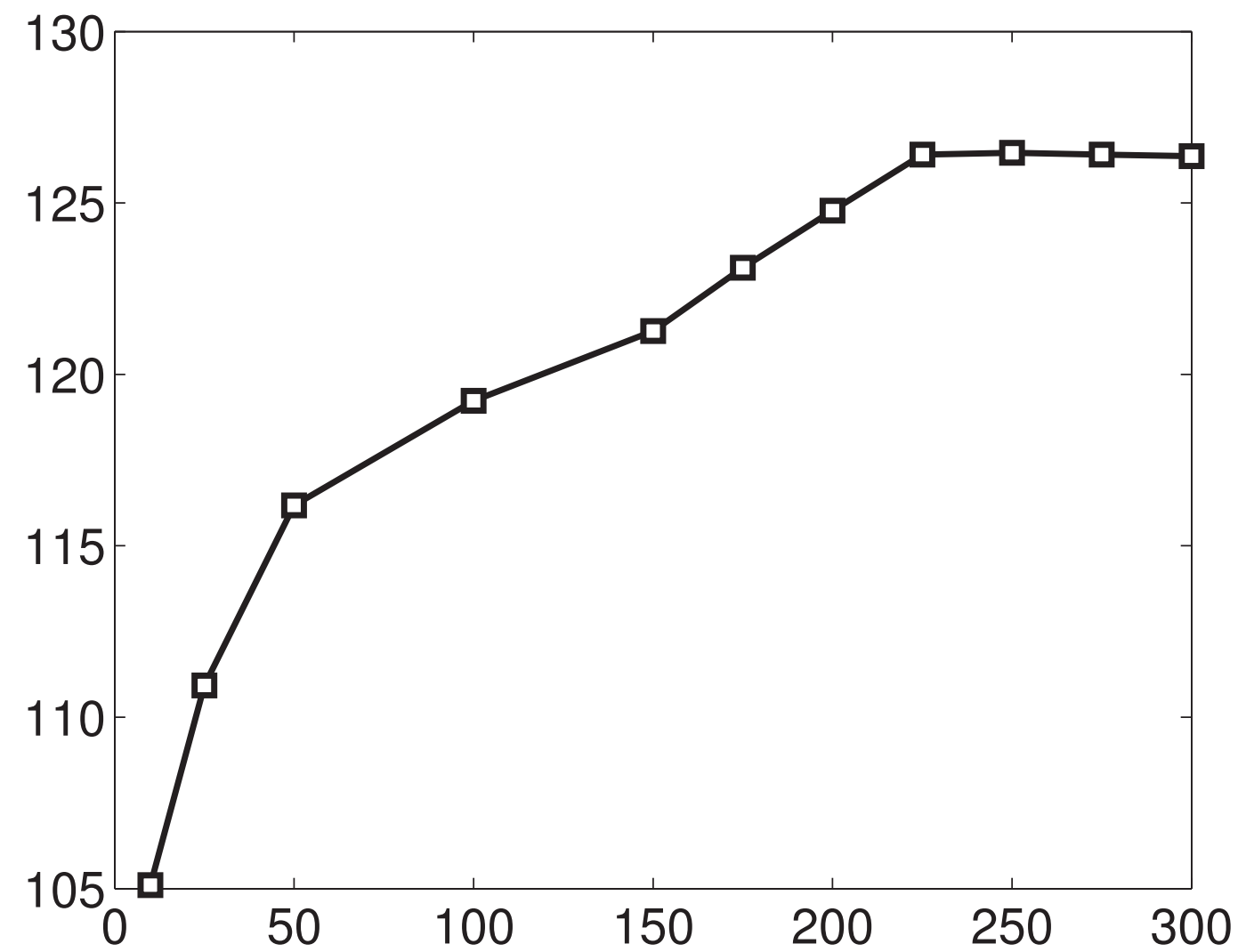

Fig. 4. Convergence analysis : Graph of function $N_{1} \mapsto 20 \log _{10}\left(\operatorname{Conv}\left(n_{s}, N_{1}\right)\right)$ for the stochastic master system with $n_{s}=300$. Horizontal axis is $N_{1}$. 


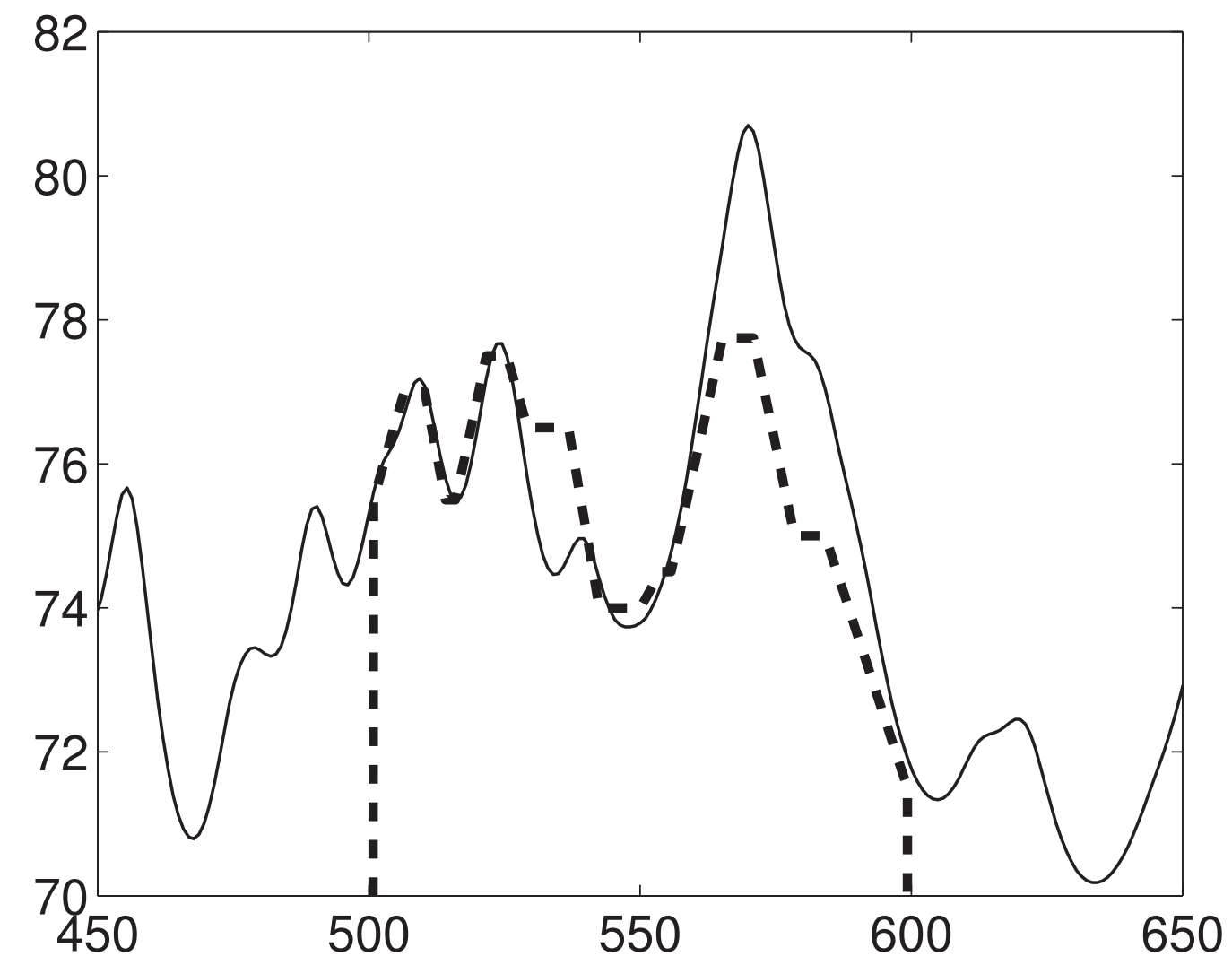

Fig. 5. Definition of the target $\nu \mapsto 20 \log _{10}(g(2 \pi \nu))$ (thick dashed line). Comparison with the reference observation $\nu \mapsto 20 \log _{10}\left(\underline{w}^{\operatorname{master}}(2 \pi \nu)\right)$ (thin solid line) in the frequency band $\mathbb{B}_{1}=[500,600] \mathrm{Hz}$ (horizontal axis). 

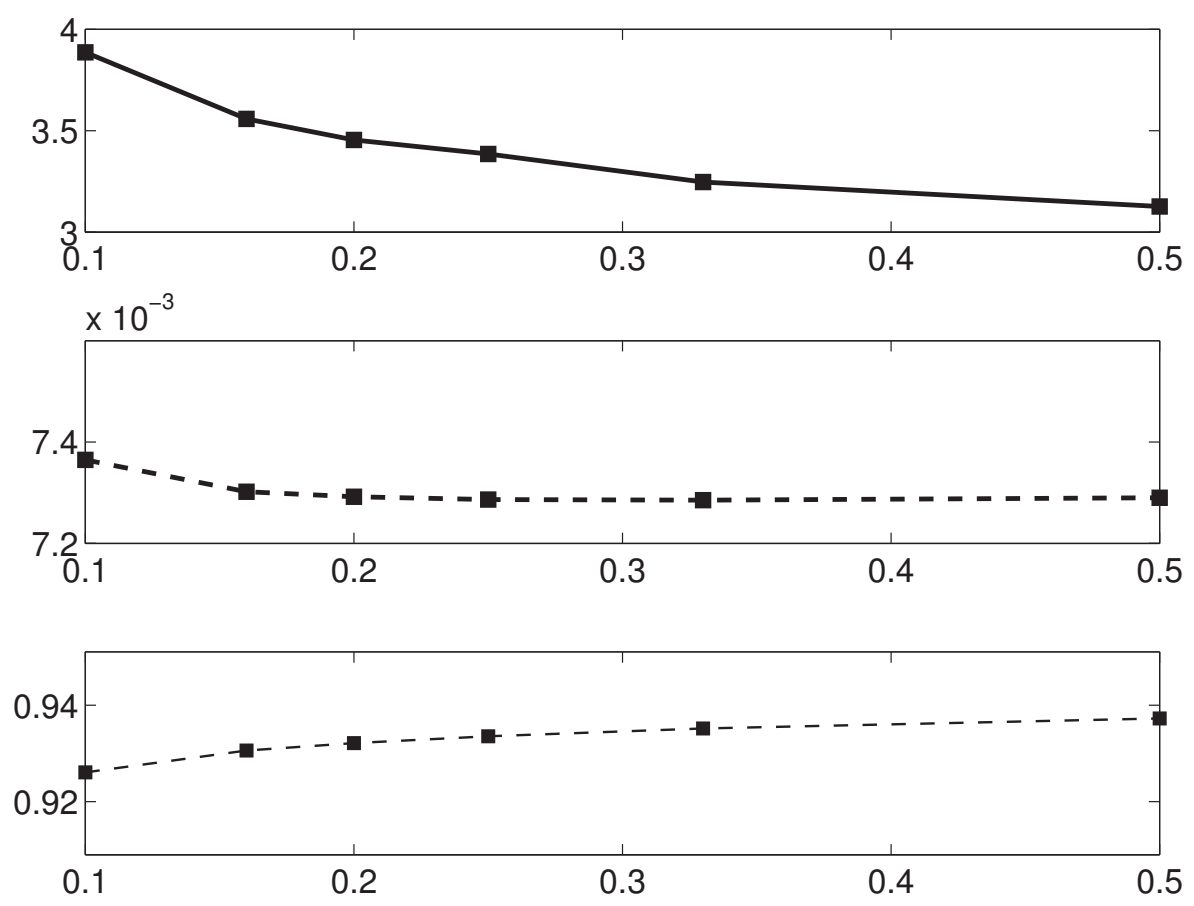

Fig. 6. Case 1. Sensitivity of the robust design optimization with respect to the weighting factor $\alpha \in] 0,0.5]$ : Graph of $\alpha \mapsto \frac{\mathrm{m}^{\mathrm{RD}}}{\mathrm{m}^{\mathrm{D}}}$ (thick line), graph of $\alpha \mapsto j\left(\mathrm{~m}^{\mathrm{RD}}, \alpha\right)$ (thick dashed line), graph of $\alpha \mapsto \frac{\left\|\sigma^{\mathrm{RD}}\right\|_{\mathbb{B}_{1}}}{\left\|\sigma^{\mathrm{D}}\right\|_{\mathbb{B}_{1}}}$ (thin dashed line). Horizontal axis is $\alpha$. 


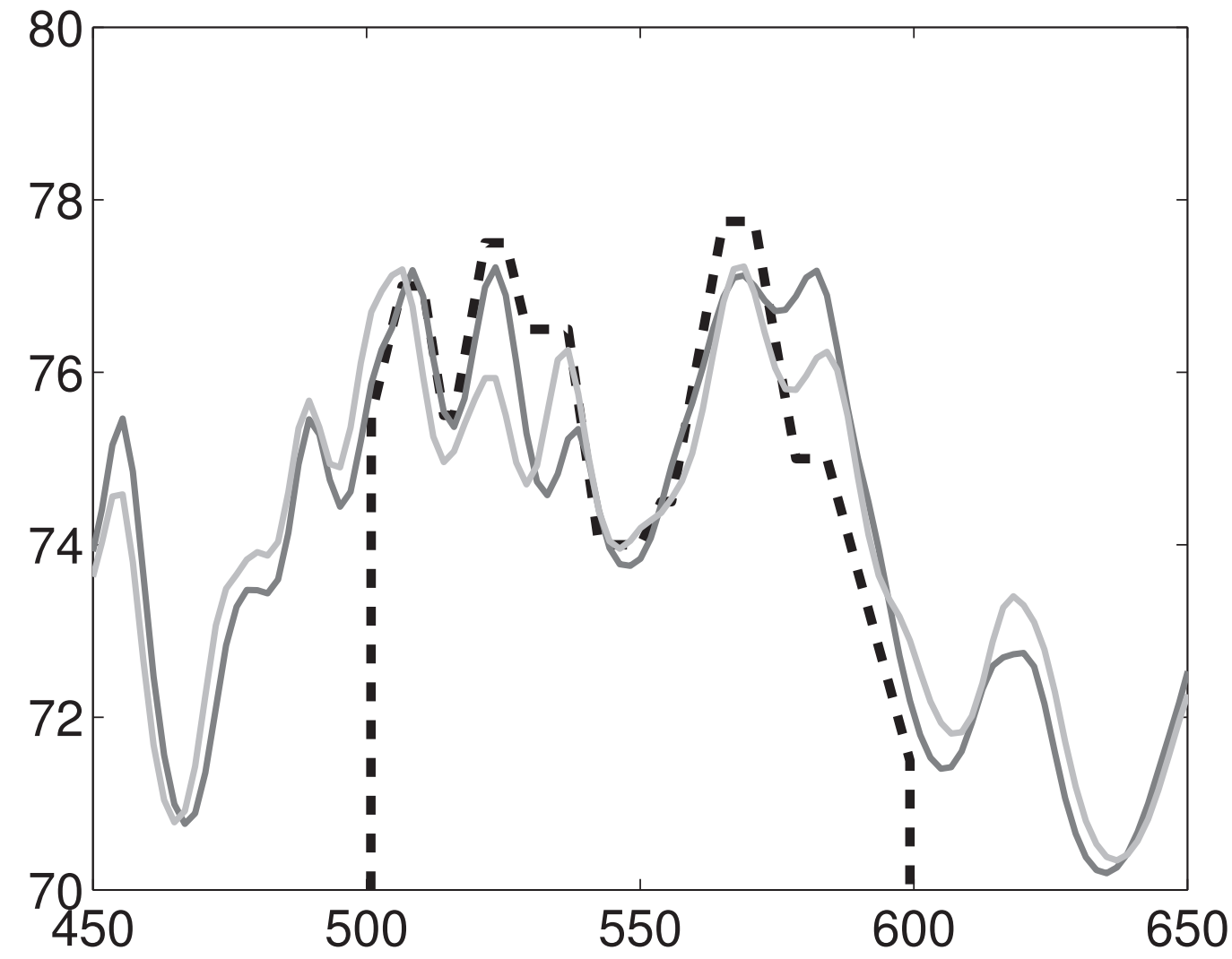

Fig. 7. Case 1. Comparison of the target $\nu \mapsto 20 \log _{10}(g(2 \pi \nu))$ (thick dashed line) with the response of the mean model corresponding to the design optimization $\nu \mapsto 20 \log _{10}\left(\underline{w}^{\mathrm{D}}(2 \pi \nu)\right)$ (thin dark gray line) and corresponding to the robust design optimization $\nu \mapsto 20 \log _{10}\left(\underline{w}^{\mathrm{RD}}(2 \pi \nu)\right)$ (thin light gray line) for $\alpha=1 / 2$. Horizontal axis is the frequency $\nu$ in $\mathrm{Hz}$. 


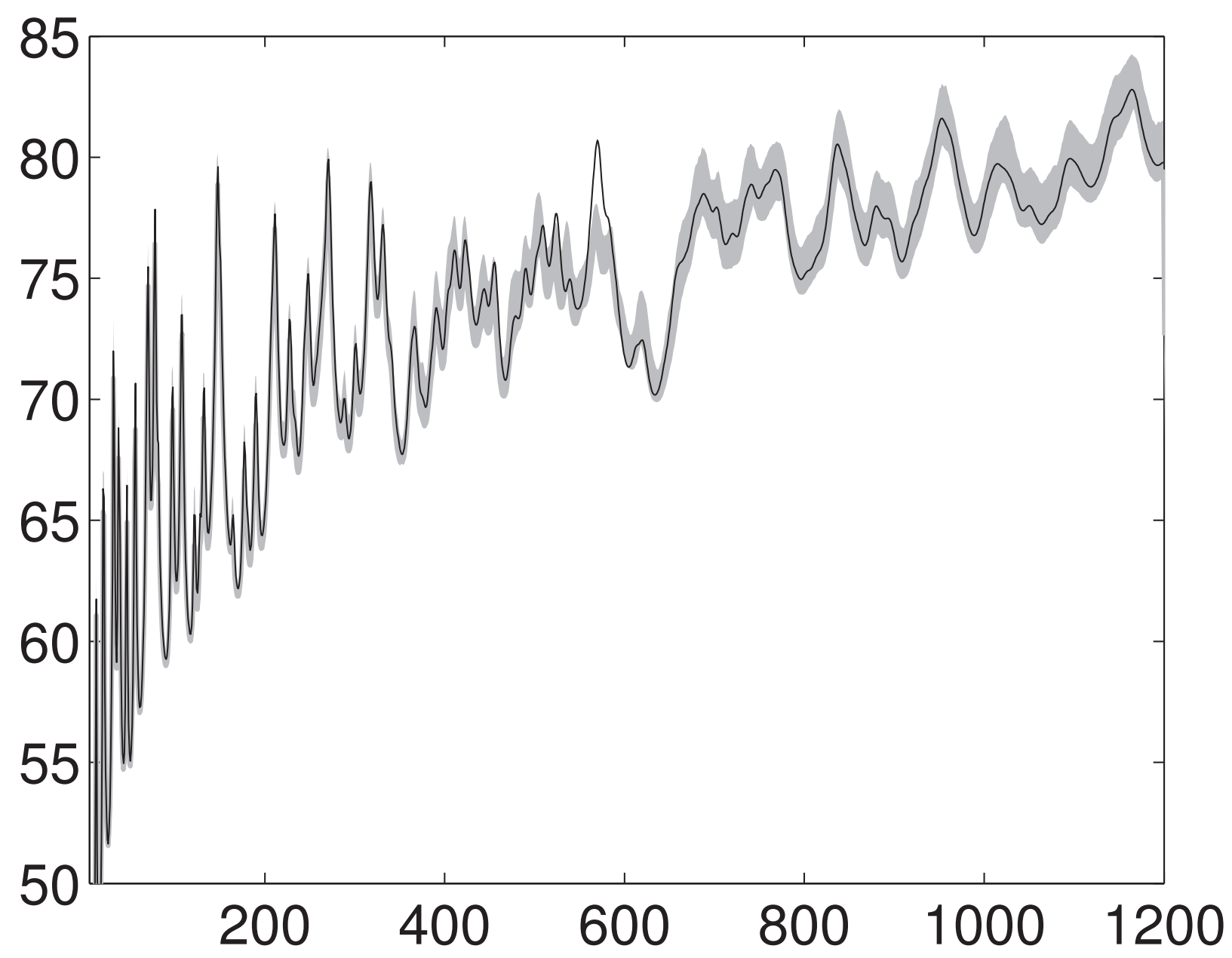

Fig. 8. Case 1. Comparison of the reference observation $\nu \mapsto 20 \log _{10}\left(\underline{w}^{\operatorname{master}}(2 \pi \nu)\right)$ (thin solid line) with the confidence region (light gray region) of random response for the robust design, over the band $\mathbb{B}=[5,1200] \mathrm{Hz}$ (horizontal axis) and for $\alpha=1 / 2$ and a probability level $P_{c}=0.95$. Horizontal axis is the frequency $\nu$ in $\mathrm{Hz}$. 

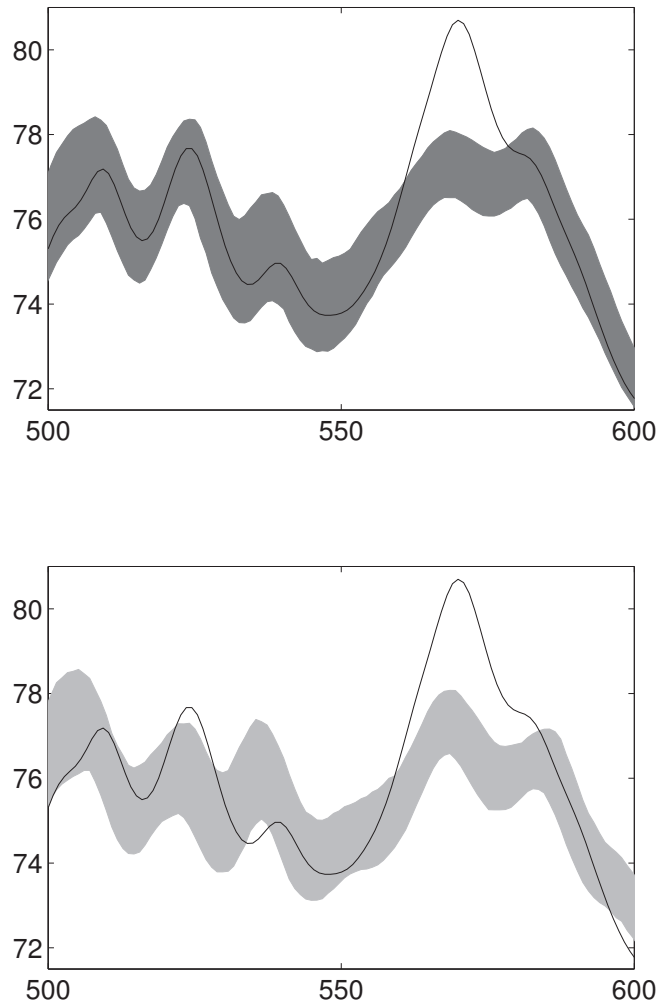

Fig. 9. Case 1. Comparison of the reference solution $\nu \mapsto 20 \log _{10}\left(\underline{w}^{\operatorname{master}}(2 \pi \nu)\right)$ (thin solid line) with the confidence region (dark gray region) of the random response $\nu \mapsto 20 \log _{10}\left(W^{\mathrm{D}}(2 \pi \nu)\right)$ corresponding to the design optimization and with the confidence region (light gray region) of the random response $\nu \mapsto 20 \log _{10}\left(W^{\mathrm{RD}}(2 \pi \nu)\right)$ corresponding to the robust design optimization. Horizontal axis is the frequency $\nu$ in $\mathrm{Hz}$. 


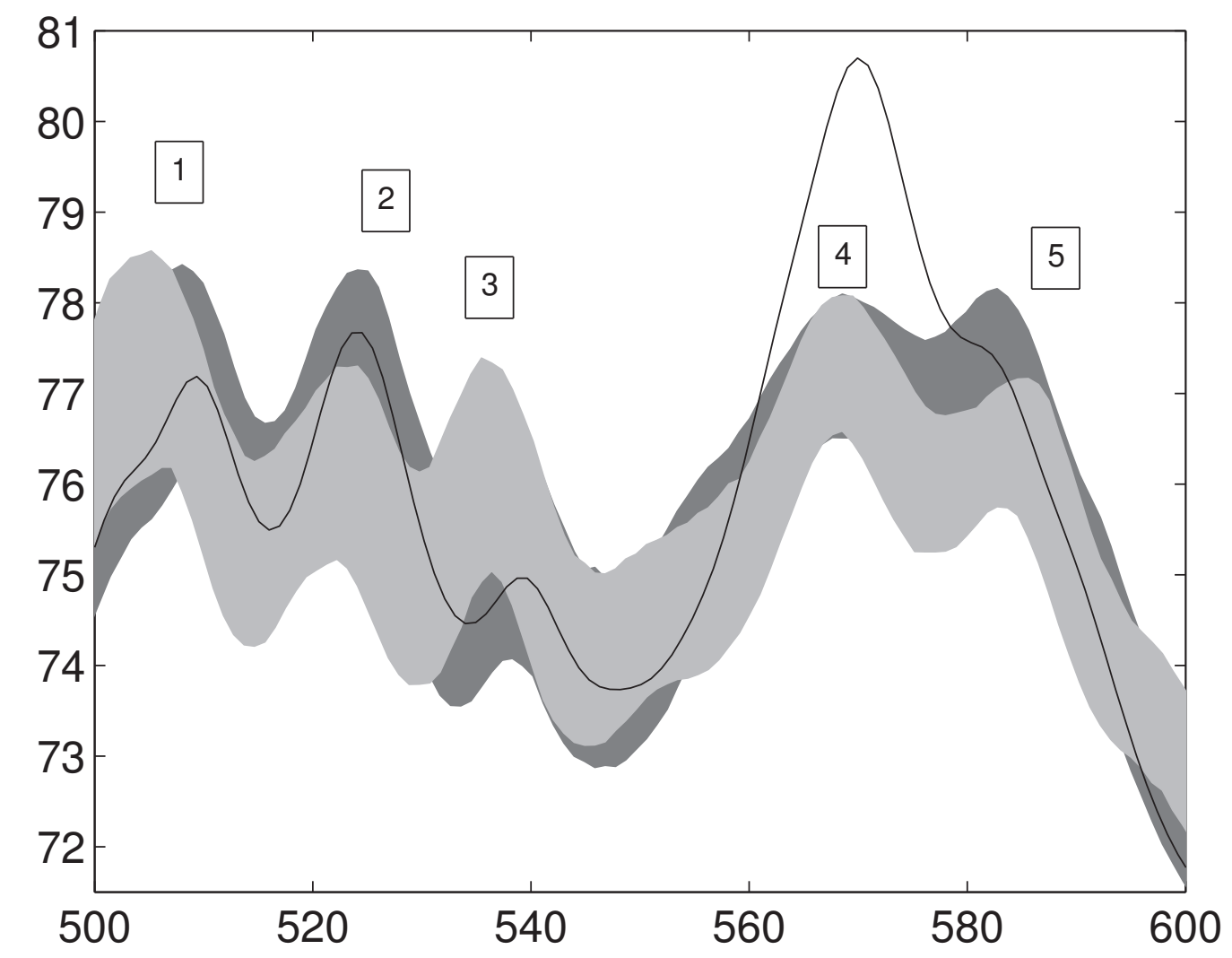

Fig. 10. Case 1. Comparison of the reference solution $\nu \mapsto 20 \log _{10}\left(\underline{w}^{\operatorname{master}}(2 \pi \nu)\right)$ (thin solid line) with the two confidence regions defined in Fig. 9. 


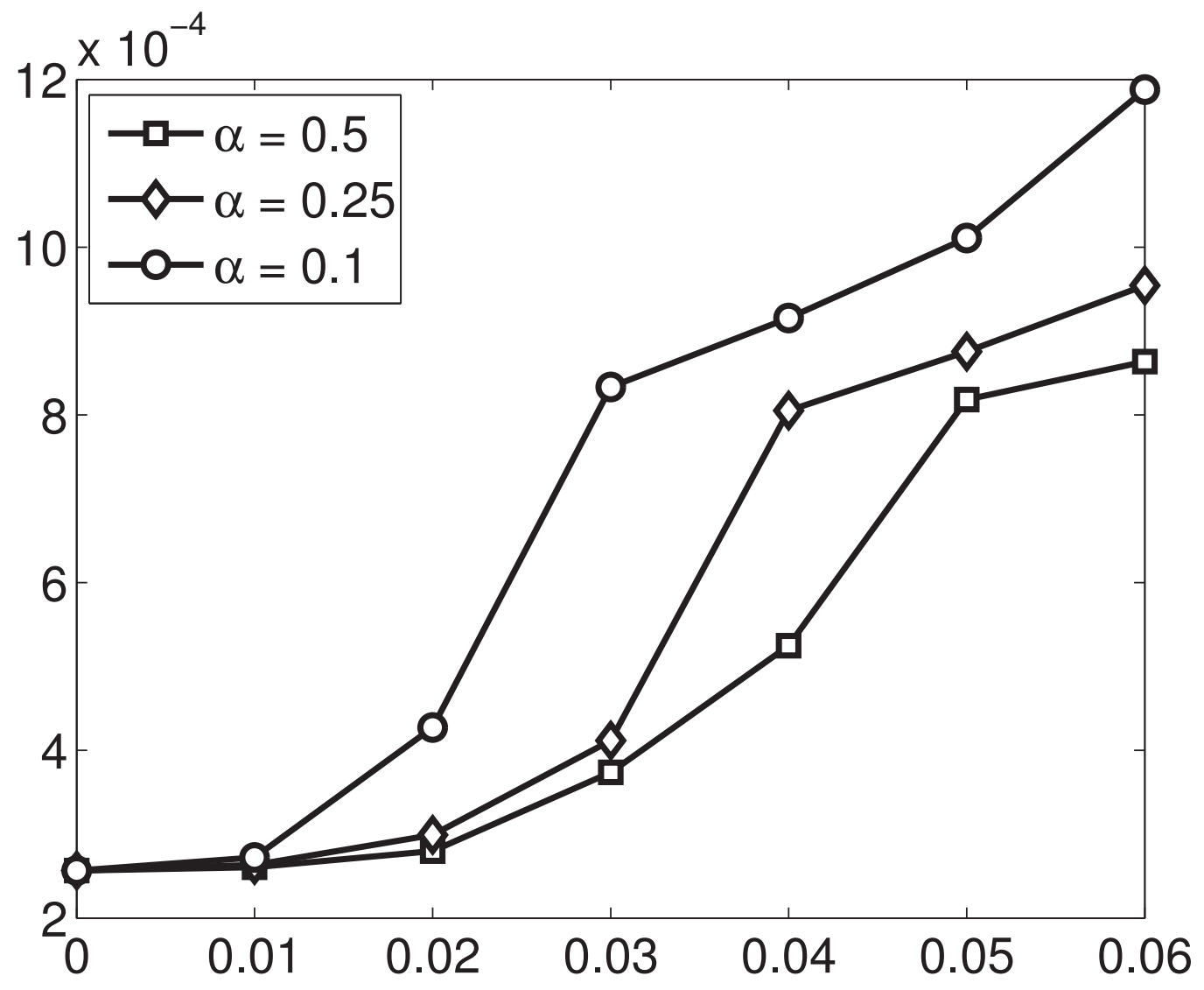

Fig. 11. Case 1. Sensitivity analysis for the robust design optimization : Graph of function $\boldsymbol{\delta} \mapsto \mathrm{m}^{\mathrm{RD}}(\boldsymbol{\delta})$ for $\alpha=0.5$ (thin line with $\square$ ), for $\alpha=0.25$ (thin line with $\diamond$ ), for $\alpha=0.1$ (thin line with $\circ$ ). Horizontal axis is $\boldsymbol{\delta}$. 


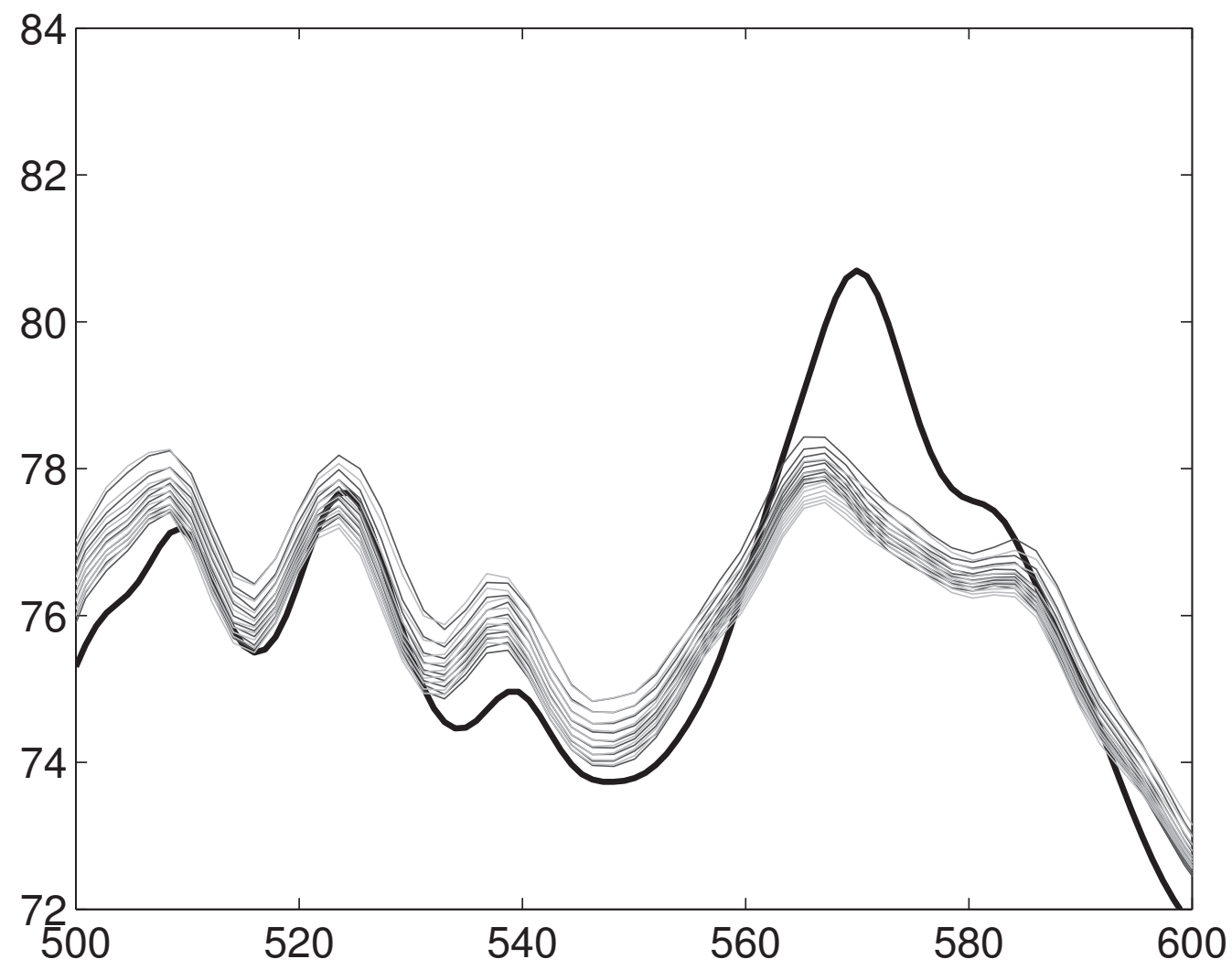

Fig. 12. Case 2, low uncertainty level. Comparison of the reference solution $\nu \mapsto 20 \log _{10}\left(\underline{w}^{\operatorname{master}}(2 \pi \nu)\right)$ (thick solid line) with the $10,20,30,40,50,60,70,80,90$ th quantiles of the random response $\nu \mapsto 20 \log _{10}\left(W^{\mathrm{D}}(2 \pi \nu)\right)$ (thin black lines) corresponding to the design optimization and of the random response $\nu \mapsto 20 \log _{10}\left(W^{\mathrm{RD}}(2 \pi \nu)\right)$ (thin gray lines) corresponding to the robust design optimization. Horizontal axis is the frequency $\nu$ in $\mathrm{Hz}$. 


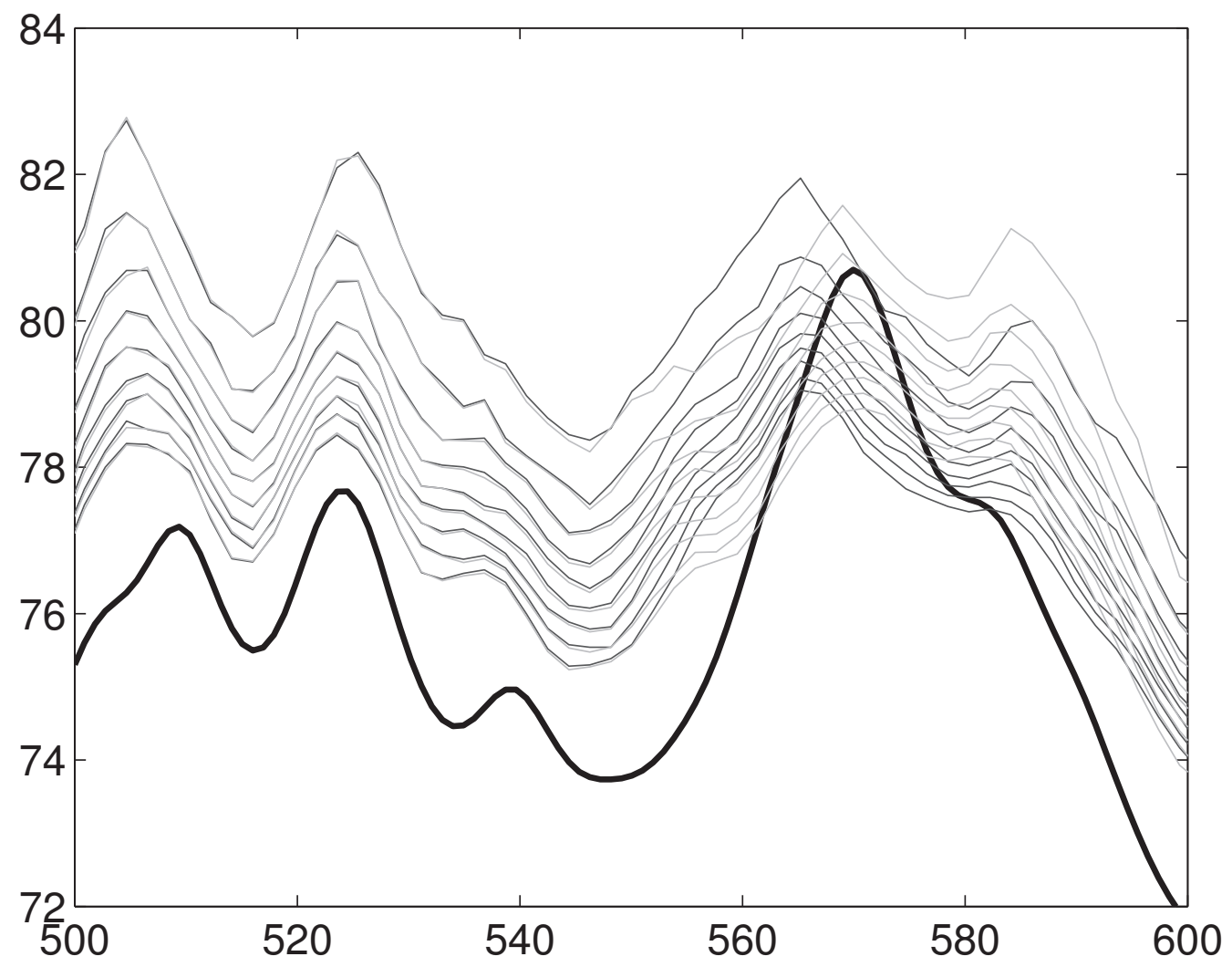

Fig. 13. Case 2, medium uncertainty level. Comparison of the reference solution $\nu \mapsto 20 \log _{10}\left(\underline{w}^{\operatorname{master}}(2 \pi \nu)\right)$ (thick solid line) with the $10,20,30,40,50,60,70,80,90$ th quantiles of the random response $\nu \mapsto 20 \log _{10}\left(W^{\mathrm{D}}(2 \pi \nu)\right)$ (thin black lines) corresponding to the design optimization and of the random response $\nu \mapsto 20 \log _{10}\left(W^{\mathrm{RD}}(2 \pi \nu)\right)$ (thin gray lines) corresponding to the robust design optimization. Horizontal axis is the frequency $\nu$ in $\mathrm{Hz}$. 